Provided for non-commercial research and education use. Not for reproduction, distribution or commercial use.

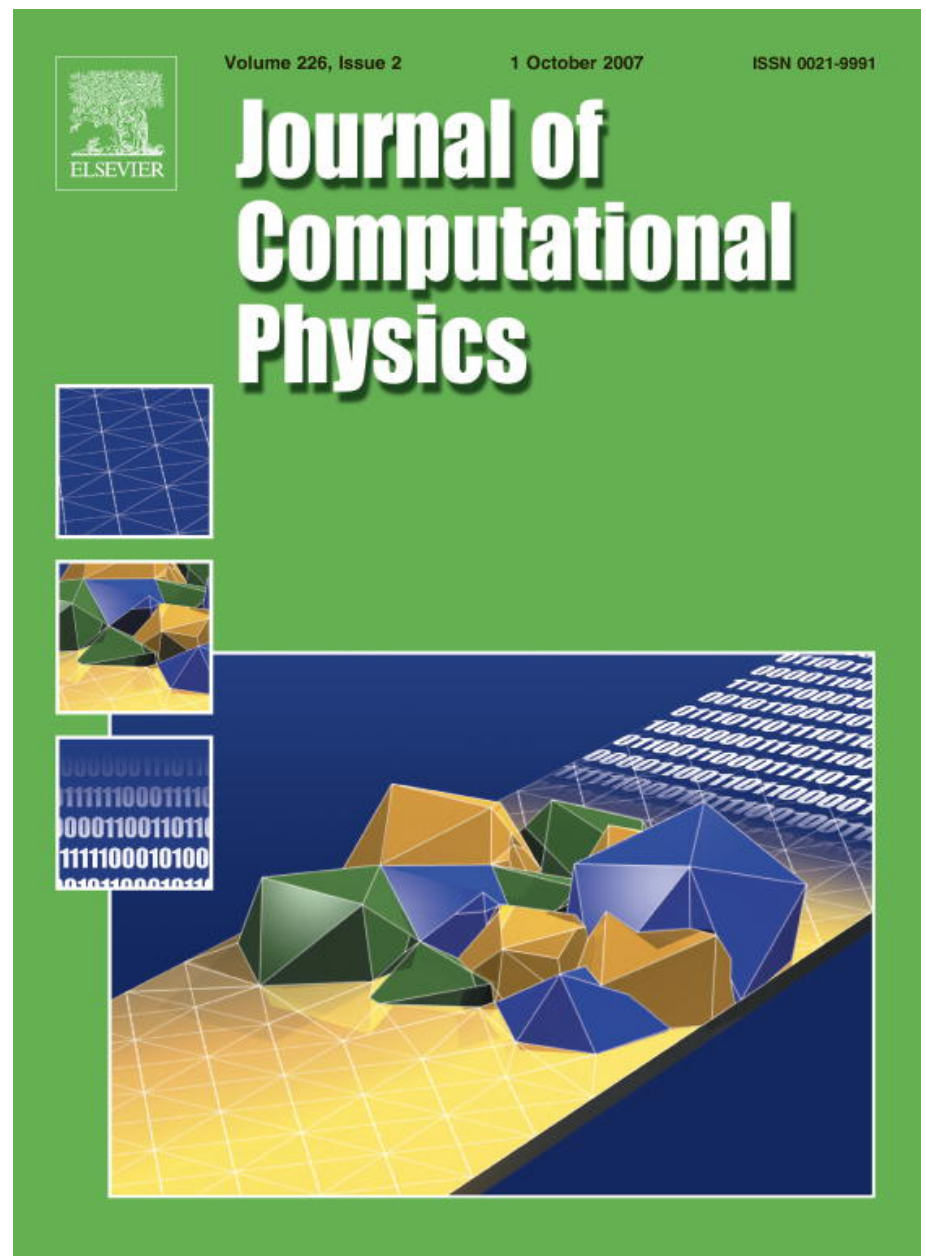

This article was published in an Elsevier journal. The attached copy

is furnished to the author for non-commercial research and education use, including for instruction at the author's institution, sharing with colleagues and providing to institution administration.

Other uses, including reproduction and distribution, or selling or licensing copies, or posting to personal, institutional or third party websites are prohibited.

In most cases authors are permitted to post their version of the article (e.g. in Word or Tex form) to their personal website or institutional repository. Authors requiring further information regarding Elsevier's archiving and manuscript policies are encouraged to visit: 


\title{
Parametric uncertainty analysis of pulse wave propagation in a model of a human arterial network
}

\author{
Dongbin Xiu $^{\mathrm{a}, *}$, Spencer J. Sherwin ${ }^{\mathrm{b}}$ \\ a Department of Mathematics, Purdue University, West Lafayette, IN 47907, United States \\ ${ }^{\mathrm{b}}$ Department of Aeronautics, South Kensington Campus, Imperial College London, SW7 2AZ, UK
}

Received 12 January 2007; received in revised form 23 April 2007; accepted 25 May 2007

Available online 8 June 2007

\begin{abstract}
Reduced models of human arterial networks are an efficient approach to analyze quantitative macroscopic features of human arterial flows. The justification for such models typically arise due to the significantly long wavelength associated with the system in comparison to the lengths of arteries in the networks. Although these types of models have been employed extensively and many issues associated with their implementations have been widely researched, the issue of data uncertainty has received comparatively little attention. Similar to many biological systems, a large amount of uncertainty exists in the value of the parameters associated with the models. Clearly reliable assessment of the system behaviour cannot be made unless the effect of such data uncertainty is quantified.

In this paper we present a study of parametric data uncertainty in reduced modelling of human arterial networks which is governed by a hyperbolic system. The uncertain parameters are modelled as random variables and the governing equations for the arterial network therefore become stochastic. This type stochastic hyperbolic systems have not been previously systematically studied due to the difficulties introduced by the uncertainty such as a potential change in the mathematical character of the system and imposing boundary conditions. We demonstrate how the application of a high-order stochastic collocation method based on the generalized polynomial chaos expansion, combined with a discontinuous Galerkin spectral $/ h p$ element discretization in physical space, can successfully simulate this type of hyperbolic system subject to uncertain inputs with bounds. Building upon a numerical study of propagation of uncertainty and sensitivity in a simplified model with a single bifurcation, a systematical parameter sensitivity analysis is conducted on the wave dynamics in a multiple bifurcating human arterial network. Using the physical understanding of the dynamics of pulse waves in these types of networks we are able to provide an insight into the results of the stochastic simulations, thereby demonstrating the effects of uncertainty in physiologically accurate human arterial networks.
\end{abstract}

(C) 2007 Elsevier Inc. All rights reserved.

Keywords: Mathematical biology; Hemodynamics; Arterial network; Stochastic modelling; Uncertainty analysis; High-order methods

\footnotetext{
* Corresponding author. Tel.: +1 7654962846.

E-mail addresses: dxiu@math.purdue.edu, dxiu@purdue.edu (D. Xiu), s.sherwin@imperial.ac.uk (S.J. Sherwin).
} 


\section{Introduction}

\subsection{Reduced modelling of arterial networks}

The one-dimensional modelling of the human arterial system was introduced by Euler in 1775 [8] who derived the partial differential equations expressing the conservation of mass and momentum for inviscid flow. In order to close the problem, he also suggested two possible, but experimentally unrealistic, constitutive equations describing the behaviour of the elastic wall with changes in the lumenal pressure. Apparently, he did not recognize the wave-like nature of the flow and therefore could not solve the equations. The wave nature of the arterial flow was, however, first described scientifically by Young [41] who derived the wave speed using an argument based on intuition and analogy to Newton's theory of the speed of sound in air. In 1877, Moens [23] and Kortweg [20] independently published analyses of flow in thin-walled elastic vessels, deriving what is now known as the Moens-Kortweg equation for the wave speed. Riemann [25] provided the analytical tools for the general equations when he introduced the method of characteristics, which was first applied to arterial flow more than 50 years ago, most notably by Anliker and co-workers [30,31] and Skalak [27].

The equations derived by Euler are a system of non-linear partial differential equations analogous to the shallow-water equations of hydrodynamics or the one-dimensional inviscid equations of gas dynamics. Typical wave-speeds in the arterial system are of the order of 3-10 m/s. Since a typically heart beat is of order of a second, linearized analysis suggests that the wavelengths of pulses in the arterial tree are of the order of 3-10 m which is far larger than any length of individual artery. Therefore, under physiological/healthy conditions of the human arterial system, the equations are only weakly non-linear and therefore many characteristics of the flow can be captured by the linearized system. This is essentially the approach of Womersley [35] who linearized the two-dimensional equations for flow in straight, circular elastic tubes and obtained the wave solution by Fourier techniques. This linear analysis has become the "standard" model of waves in the arteries found in most hemodynamics textbooks. The success of the linearized model and the apparently periodic nature of the arterial system has led most investigators since Womersley to analyze arterial flow in the frequency rather than the time domain using the so-called "electrical" analogy.

Although there is a large body of work using the frequency-domain analysis, many facets of the physiological waveforms have yet to be explained. Direct analysis and modelling of the hyperbolic system in the timedomain allows various advantages permitting the investigator to understand more of the physical properties of the system [4,5]. For example, an assumption used in the frequency-domain approach is that the arterial system is in a state of "steady oscillation". This is not always true, since ectopic beats arise when the contraction of the heart is blocked or is so ineffective that the aortic valve never opens. In this case, neither the flow nor the pressure shows any hint of the previous periodic behaviour. However, this effect can be understood from analysis of the time-domain hyperbolic system since the characteristic speed of wave propagation is sufficiently fast that the time scale to propagate information through the whole arterial system is much smaller than the duration of the cardiac cycle.

Although the system is only weakly non-linear, the bifurcating network of arteries in the network leads to multiple reflections leading to relatively complicated pulse wave patterns. The need to propagate the waves over many wavelengths without numerical dispersion makes the modelling of the system relatively challenging. Time-domain analysis was investigated by Wang and Parker [33,34] who adopted a semi-analytical time-space domain approach to model the linearized wave motion in arteries. The method of characteristic has also previously been applied by Skalak [27], Stettler et al. [30,31] and Stergiopulos et al. [29]. More recently aeronautical-type numerical modelling techniques have been applied to the problem (e.g. $[26,10])$.

\subsection{Stochastic modelling}

While modelling of pulse wave propagation in the human arterial system received considerable amount of attention, little has been paid to the effects of uncertainty in the models. In complex biological fields, mathematical models serve as simplified and reduced representations of the true physics and, although useful, their usage is often constrained by the difficulty of assigning numerical values to various parameters and boundary/ initial conditions required by the governing equations. Furthermore, the complexity of the physics, e.g., large 
variability in human body conditions, results in a significant amount of uncertainty associated with these "parameters"- the so-called parametric uncertainty. Traditional approach assigns "most likely" values to the parameters, via some parameter estimation procedures, and such an approach could be inadequate as the complex biological processes may depend sensitively to some of the parameters. Also, very often observables from experimental measurements are not repeated enough times for reliable statistical estimates to be made on the "likelihood" of the parameter values. Therefore, mathematical and numerical techniques are needed to develop effective means of quantifying parameter uncertainty and its effect in complex biological systems.

One of the most commonly used methods for uncertainty analysis is the Monte Carlo sampling (MCS). Although MCS is straightforward to apply as it only requires repetitive executions of deterministic simulations, typically a large number of such executions are needed as the solution statistics converge relatively slowly, e.g., the mean value typically converges as $1 / \sqrt{K}$ where $K$ is the number of realizations (e.g., [9]). The need for large number of realizations for accurate results can incur excessive computational burden, especially for systems that are already computationally expensive in their deterministic settings.

A framework of generalized polynomial chaos (gPC) [39] was recently developed and it offers possibility of developing fast numerical algorithms for uncertainty analysis in complex systems. The gPC framework extends the earlier polynomial chaos (PC) method, which was first pioneered by Ghanem and co-workers and successfully applied to many problems (cf. [17,14,16,15]). With gPC, stochastic quantities are expressed as general orthogonal polynomials of the input random parameters, and different types of orthogonal polynomials can be chosen to achieve better convergence $[39,40]$. The original PC approach by Ghanem, which employs Hermite polynomials, can be naturally incorporated in the gPC framework. A gPC expansion is essentially a spectral representation in random space, and exhibits fast convergence when the expanded function depends smoothly on the random parameters. Exponentially fast convergence can be achieved under certain circumstances. (See $[7,39,40,38]$ for detailed discussions.)

When applied to differential equations with random inputs, the quantities to be solved are the expansion coefficients of the gPC expansion. A typical approach is to conduct a Galerkin projection to minimize the error of the finite-order gPC expansion, and the resulting set of equations for the expansion coefficients are deterministic and can be solved via conventional numerical techniques. This has been done in various applications and proved to be very effective (cf. $[1,7,11,17,21,39,40,38])$.

Stochastic Galerkin (SG) procedure can be challenging when the governing stochastic equations take complicated forms, in which case the derivation of the equations for the gPC coefficients can be very difficult, if not impossible. To this end, high-order stochastic collocation (SC) approach can be used $[6,37,36]$. While retaining the high accuracy of gPC approximation, the implementation of a SC algorithm is straightforward, as it only requires repetitive realizations of a deterministic solver - similar to MCS. The choice of the realizations points - "sampling points"- is where SC differs from MCS. (Indeed MCS is a type of collocation method on random points, and there has been an attempt to utilize it to speed up PC computations [13].) Earlier engineering applications use tensor products of one-dimensional quadrature points and are not proper for problems with large number of random variables because of the exponential growth of the number of points (cf. [32]). Although a recent study for stochastic elliptic equations suggests that the tensor product approach is of high order, typically the number of random variables is restricted to less than five in practical applications [6]. On the other hand, the work of $[37,36]$ represents a systematical attempt to avoid using tensor product points. Specifically, the so-called "sparse grid" [28] is found to be highly efficient in handling problems with random variables of as many as 50 [37]. Error bound and examples of problems with complicated governing equations can be found in [36], and applications to random natural convection problems with adaptive choice of sparse grid can be found in [12].

\subsection{Outline of paper}

The gPC framework serves as a solid foundation to study problems with parametric uncertainty of large variability. This is particularly true for biological problems, where tremendous amount of data uncertainty exists due to lack of accurate measurement and/or large variation in the underlying systems. A straightforward application of stochastic Galerkin approach is challenging because of the complex form of the governing 
equations. In fact, no systematical numerical study has been conducted for stochastic hyperbolic systems such as the one considered in this paper. (The study in [18] demonstrated that even for the simplest hyperbolic problem, a scalar wave equation, uncertainty can change the properties of the stochastic Galerkin equations significantly.) Here the pseudo-spectral type stochastic collocation method presented in [36] is employed to study the effect of parametric uncertainty in a human arterial network model. We first present the mathematical framework for such a model, in both deterministic and stochastic settings. We then briefly introduce the gPC expansion and stochastic collocation method. The method is applied to two examples for which we also analyze parametric sensitivity. One is a simplified model case with one-bifurcation, where we study the uncertainty propagation and evolution of sensitivity coefficients. The other is a full scale network for a human arterial system containing 37 branches and it represents the first such study on uncertainty and data sensitivity in physiologically realistic simulations.

\section{Methodology}

\subsection{Deterministic model for human artery network}

\subsubsection{Governing deterministic equations}

The mathematical model is based on the non-linear, one-dimensional equations of pressure and flow wave propagation in compliant vessels. The governing system of equations results from conservation of mass and momentum applied to a one-dimensional impermeable and deformable tubular control volume of incompressible and Newtonian fluid. They take the form [26]

$$
\begin{aligned}
& \frac{\partial A}{\partial t}+\frac{\partial(A U)}{\partial x}=0 \\
& \frac{\partial U}{\partial t}+U \frac{\partial U}{\partial x}=-\frac{1}{\rho} \frac{\partial p}{\partial x}+\frac{f}{\rho A},
\end{aligned}
$$

where $x$ is the axial coordinate along the vessel, $t$ is the time, $A(x, t)$ is the cross-sectional area of the vessel, $U(x, t)$ is the average axial velocity, $p(x, t)$ is the average internal pressure over the cross-section, $\rho$ is the density of blood and $f(x, t)$ is the friction force per unit length. In what follows we will concentrate on the inviscid limit where $f=0$ typically associated with the larger conduit arteries in the cardiovascular system.

The system of equations is completed with a pressure-area relation, $p=\mathcal{F}(A ; x, t)$. We will adopt a relation previously used in $[34,26,24,4,5]$ which assumes a thin, homogeneous and elastic arterial wall and it takes the form

$$
p=p_{0}+\beta\left(\sqrt{A}-\sqrt{A_{0}}\right), \quad \beta=\frac{\sqrt{\pi} h E}{\left(1-\sigma^{2}\right) A_{0}},
$$

where $A_{0}(x)$ and $h(x)$ are the sectional area and wall thickness at the reference state $\left(p_{0}, U_{0}\right)$, with $p_{0}$ and $U_{0}$ assumed to be zero, $E(x)$ is the Young's modulus and $\sigma$ is the Poisson's ratio, typically taken to be $\sigma=1 / 2$ since biological tissue is practically incompressible. Whilst the pressure-area relationship is non-linear with the property that at higher pressures the arteries are more stiff, the normal physiological/healthy range can reasonably be approximately as linear in terms of the radius as suggested by Eq. (3). Using Eq. (3) the parameter $\beta$ can be directly related to the speed of pulse wave propagation, $c$, through [26]

$$
c=\sqrt{\frac{\beta}{2 \rho}} A^{1 / 4} .
$$

\subsubsection{The characteristic system}

Following the work of $[10,30,33]$, we consider Eqs. (1) and (2) with the pressure-area relationship (3), and $\beta, A_{0}$ constant. We write the system in non-conservative form as

$$
\frac{\partial \boldsymbol{U}}{\partial t}+\boldsymbol{H}(\boldsymbol{U}) \frac{\partial \boldsymbol{U}}{\partial x}=0
$$


where

$$
\boldsymbol{U}=\left[\begin{array}{l}
A \\
U
\end{array}\right] \quad \boldsymbol{H}=\left[\begin{array}{cc}
U & A \\
\frac{1}{\rho D A} & U
\end{array}\right]
$$

and we have introduced the distensibility $D$ defined as $D=\frac{1}{A} \frac{\mathrm{d} A}{\mathrm{~d} p}$.

Under the assumption that $A>0$, indeed a necessary condition to have a physically relevant solution, the matrix $\boldsymbol{H}$ has two real eigenvalues

$$
\lambda_{1,2}(\boldsymbol{H})=U \pm c,
$$

where $c=\frac{1}{\sqrt{\rho D}}$ is the wave speed for the non-linear system. For typical values of velocity, vessel area and the elastic parameter $\beta$ encountered in arteries under normal physiological conditions, we find that the system is subsonic and so $\lambda_{1}>0$ and $\lambda_{2}<0$. Next we introduce a change of variables [26] and transform Eq. (5) into its characteristic system of the form

$$
\frac{\partial \boldsymbol{W}}{\partial t}+\boldsymbol{\Lambda} \frac{\partial \boldsymbol{W}}{\partial x}=\mathbf{0}
$$

where $\boldsymbol{\Lambda}$ is a diagonal matrix containing the eigenvalues and the characteristic variables are defined as

$$
W_{1,2}=\int_{U_{0}}^{U} \mathrm{~d} U \pm \int_{A_{0}}^{A} \frac{c}{A} \mathrm{~d} A=U-U_{0} \pm \int_{A_{0}}^{A} \frac{c}{A} \mathrm{~d} A,
$$

where $\left(U_{0}, A_{0}\right)$ is taken as a reference state. (The characteristic variables given by Eq. (6) are also called the Riemann invariants of the system (1) and (2) in terms of the $(A, u)$ variables).

Finally, for the pressure area relationship defined in Eq. (3) we can derive an explicit form of $\boldsymbol{W}$. Recalling that $c=1 / \sqrt{\rho D}$ and evaluating $D$ for the pressure area relationship (3), we obtain

$$
W_{1,2}=U \pm 4\left(c-c_{0}\right)=U \pm 4 \sqrt{\frac{\beta}{2 \rho}}\left(A^{1 / 4}-A_{0}^{1 / 4}\right),
$$

where we have assumed that $U_{0}=0$ when $A=A_{0}$. The above expression was also obtained by Formaggia et al. [10] who considered the more general $(A, Q)$ system.

\subsubsection{Numerical scheme}

Eqs. (1)-(3) are solved using a discontinuous Galerkin scheme with a spectral/hp spatial discretization and a second-order Adams-Bashforth time-integration scheme. This scheme is suitable for the 1D formulation because it can propagate waves of different frequencies without suffering from excessive dispersion and diffusion errors. Details on this algorithm can be found in $[19,26]$.

\subsubsection{Treatment of boundary conditions and bifurcations}

To close the system we require boundary conditions for incoming characteristics and also a condition to couple multiple segments into an arterial network of bifurcating vessels. Since full details have previously been discuss in [26], here we highlight the main implementation points in what follows.

The choice of an explicit time-integration scheme in our numerical discretization allows boundary conditions of the hyperbolic system to be enforced using characteristic information at an explicit time level. Therefore at an inflow boundary of an arterial network we use the solution of a local Riemann problem to enforce either a known value of the pressure or velocity in time. Alternatively, we can impose an absorbing boundary where a desired incoming state is weakly enforced through the upwind characteristic information whilst the outgoing characteristic remains constant.

Finally, to enable a network of many bifurcating arteries to be linked we need an interface condition at a bifurcation as illustrated in Fig. 1. Looking at the problem from the characteristic point of view, information can only reach the bifurcation from within vessel 1 by a forward travelling wave. The forward travelling wave is governed in terms of the characteristic variable $W_{1}^{1}$ (where superscript denotes the vessel number) and according to Eq. (7) is a function of $\left(A_{1}, U_{1}\right)$. Similarly within the daughter vessels information can only reach the bifurcation by a backwards travelling wave which is governed by characteristic variables $W_{2}^{2}\left(A_{2}, U_{2}\right)$ or 


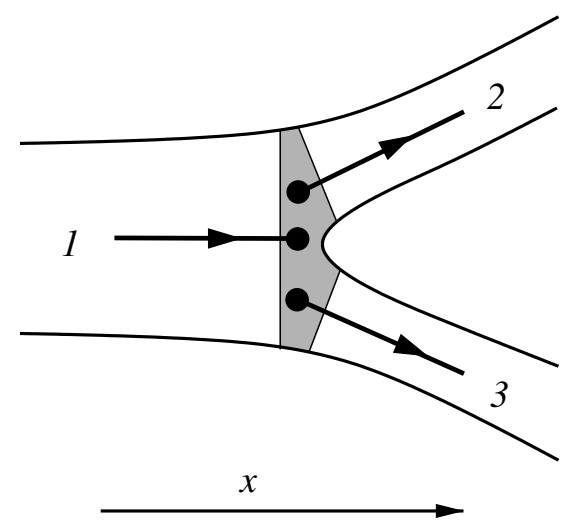

Fig. 1. Notation for arterial tree bifurcation.

$W_{2}^{3}\left(A_{3}, U_{3}\right)$. The hyperbolic nature of the problem reduces the incoming information to three constant characteristic variables $W_{1}^{1}, W_{2}^{2}$ and $W_{2}^{3}$. The first three equations of the Riemann problem are obtained by imposing that the characteristic variables in each vessel should remain constant. From the definition of the characteristic variables for the non-linear system, given by Eq. (7), we have

$$
\begin{aligned}
& U_{1}+4\left(c_{1}-c_{0}^{1}\right)=W_{1}^{1}, \\
& U_{2}-4\left(c_{2}-c_{0}^{2}\right)=W_{2}^{2}, \\
& U_{3}-4\left(c_{3}-c_{0}^{3}\right)=W_{2}^{3},
\end{aligned}
$$

where $c_{0}^{1}, c_{0}^{2}, c_{0}^{3}$ are the values of the wave speed $c$ evaluated using the equilibrium area $A_{0}$ in vessels 1,2 and 3 , respectively.

To close the problem we require another three independent equations. The first condition is physically motivated by requiring that the mass is conserved through the bifurcation and, therefore, mass flux balance results in $Q_{1}=Q_{2}+Q_{3}$. The other two conditions are obtained from the requirement of continuity of the momentum flux at the bifurcation. This leads to the condition that total pressure $p+\frac{1}{2} \rho U^{2}$ should be continuous at the boundary. These requirements provide the three additional equations:

$$
\begin{aligned}
& A_{1} U_{1}=A_{2} U_{2}+A_{3} U_{3}, \\
& p_{1}+\frac{1}{2} \rho U_{1}^{2}=p_{2}+\frac{1}{2} \rho U_{2}^{2}, \\
& p_{1}+\frac{1}{2} \rho U_{1}^{2}=p_{3}+\frac{1}{2} \rho U_{3}^{2} .
\end{aligned}
$$

In summary, the six equations given by (8)-(13) define a non-linear system of algebraic equations which determine the values $\left(A_{1}, U_{1}\right),\left(A_{2}, U_{2}\right)$ and $\left(A_{3}, U_{3}\right)$ at the bifurcation. The inputs to the system are the material properties of the vessels $\beta$ or equivalently their distensibilities, $D$, the vessel equilibrium areas $A_{0}$ at the bifurcation and the values of $W_{1}^{1}, W_{2}^{2}$ and $W_{2}^{3}$ which can be evaluated from the initial values of $\left(A_{1}, U_{1}\right),\left(A_{2}, U_{2}\right)$ and $\left(A_{3}, U_{3}\right)$. The solution to this Riemann problem is used to evaluate an upwind flux at the junction in the discontinuous Galerkin numerical discretization.

\subsection{Stochastic model for the human arterial network}

This section outlines the mathematical framework for general hyperbolic systems with random inputs. In addition, we present the generalized polynomial chaos (gPC) expansion and the corresponding stochastic discretization schemes to transform the stochastic governing equations into deterministic equations.

\subsubsection{Mathematical formulations for random hyperbolic equations}

Let $x \in \mathbb{R}$ and restrict our exposition to one spatial dimension. Let $T>0$ be some fixed time, and $(\Omega, \mathcal{F}, \mathcal{P})$ be a complete probability space, where $\Omega$ is the set of outcomes, $\mathcal{F} \subset 2^{\Omega}$ the $\sigma$-algebra of events and 
$\mathcal{P}: \mathcal{F} \rightarrow[0,1]$ a probability measure. We study the following random differential equation: find a stochastic vector function, $\mathbf{v}=\left[v_{1}, \ldots, v_{m}\right]^{\mathrm{T}}: \mathbb{R} \times[0,1] \times \Omega \rightarrow \mathbb{R}^{m}$, such that for $\mathcal{P}$ - almost everywhere $\omega \in \Omega$, the following equation holds:

$$
\frac{\partial \mathbf{v}}{\partial t}(x, t, \omega)+\frac{\partial \mathbf{F}(\mathbf{v})}{\partial x}(x, t, \omega)=\mathbf{s}(x, t, \omega), \quad \text { in } \mathbb{R} \times(0, T] \times \Omega,
$$

subject to the initial condition

$$
\mathbf{v}=\mathbf{g} \quad \text { on } \mathbb{R} \times\{t=0\} \times \Omega,
$$

where $\mathbf{s}, \mathbf{F}: \mathbb{R} \times[0, T] \times \Omega \rightarrow \mathbb{R}^{m}$ and $\mathbf{g}(x, \omega): \mathbb{R} \times \Omega \rightarrow \mathbb{R}^{m}$ are known stochastic functions with the following assumptions:

- $\mathbf{H} \equiv \partial \mathbf{F} / \partial \mathbf{v}: \mathbb{R} \times[0, T] \times \Omega \rightarrow \mathbb{M}^{m \times m}$ exists $\mathcal{P}$-a.e. in $\Omega$;

- $\mathbf{H}$ is strictly hyperbolic $\mathcal{P}$-a.e. in $\Omega$, i.e., for $\mathcal{P}$-a.e. $\omega \in \Omega$, the matrix $\mathbf{H}(\cdot, \omega)$ has $m$ real distinct eigenvalues;

- $\mathbf{H}(\cdot, \omega) \in C^{2}\left(\mathbb{R} \times[0, T] ; \mathbb{M}^{m \times m}\right)$ and $\sup _{\mathbb{R} \times[0, T]}\left(|\mathbf{H}(\cdot, \omega)|,\left|D_{x, t} \mathbf{H}(\cdot, \omega)\right|,\left|D_{x, t}^{2} \mathbf{H}(\cdot, \omega)\right|\right)<\infty$ for $\mathcal{P}$-a.e. $\omega \in \Omega$;

- $\mathbf{g} \in H^{1}\left(\mathbb{R} ; \mathbb{R}^{m}\right) \otimes L^{2}\left(\Omega ; \mathbb{R}^{m}\right)$ and $\mathbf{s} \in H^{1}\left(\mathbb{R} \times(0, T) ; \mathbb{R}^{m}\right) \otimes L^{2}\left(\Omega ; \mathbb{R}^{m}\right)$.

Here standard definitions for $C^{k}, L^{p}$ and $H^{1}$ spaces are employed. Under these conditions, Eq. (14) can be written equivalently in a non-conservative form as, for $\mathcal{P}$-a.e. $\omega \in \Omega$,

$$
\frac{\partial \mathbf{v}}{\partial t}+\mathbf{H}(\mathbf{v}) \frac{\partial \mathbf{v}}{\partial x}=\mathbf{s}, \quad \text { in } \mathbb{R} \times(0, T] \times \Omega,
$$

subject to initial condition

$$
\mathbf{v}=\mathbf{g} \quad \text { on } \mathbb{R} \times\{t=0\} \times \Omega .
$$

The regularity assumptions are direct extension of those in the deterministic theory.

\subsubsection{Modelling of random inputs}

The source of uncertainty in this study is the parameters in Eqs. (1)-(3). In particular, we will study the effects of uncertainty resulted from parameter $\beta$ (see (3)), which is a function of the material properties and initial cross-sectional area of the arterial vessel (it is also directly related to wave speed) and possesses large variability. Here we model the parameters $\beta$ in each segment as random variables that are independent from each other in different segments. We further assume that their values are bounded and can be expressed as

$$
\beta_{i}(\omega)=\left\langle\beta_{i}\right\rangle\left(1+\sigma_{i} y_{i}(\omega)\right), \quad i=1, \ldots, N,
$$

where $N$ is the total number of network segments. Inside each segment, $\left\langle\beta_{i}\right\rangle$ is the mean value, $y_{i}(\omega)$ is a random variable uniformly distributed in $(-1,1)$, and $\sigma_{i}>0$ is a real number controlling the variability of the parameter. We remark that distributions other than uniform can be readily handled and do not change the following formulation. In the current work we employ uniform distribution due to the lack of evidence suggesting otherwise.

In practice, the distributions of parameters should be estimated from experimental observations. When observations are scarce to render such estimations, care needs to be taken to make proper assumptions on the distributions. This is particularly true when distributions with unbounded support (e.g., Gaussian) are to be employed, as such distributions may change the properties of the underlying hyperbolic system. For example, consider a simple one-dimensional hyperbolic system

$$
\frac{\partial \mathbf{u}}{\partial t}+\frac{\partial \mathbf{F}(\mathbf{u})}{\partial x}=0,
$$

where

$$
\mathbf{u}=\left[u_{1}, u_{2}\right]^{\mathrm{T}}, \quad \mathbf{F}(\mathbf{u})=\left[u_{2}, \alpha^{2} u_{1}\right], \quad \alpha \neq 0 .
$$


The corresponding Jacobian is

$$
\mathbf{A}(\mathbf{u}) \equiv \frac{\partial \mathbf{F}(\mathbf{u})}{\partial \mathbf{u}}=\left[\begin{array}{cc}
0 & 1 \\
\alpha^{2} & 0
\end{array}\right]
$$

This is a strongly hyperbolic system with eigenvalues $\lambda_{1,2}= \pm \alpha$. Now assume that the system is subject to random input and the flux takes the form $\mathbf{F}(\mathbf{u})=\left[u_{2}(1+\epsilon(\omega)), \alpha^{2} u_{1}\right]$, where $\epsilon(\omega)$ is a random variable. The stochastic Jacobian becomes

$$
\mathbf{A}(\mathbf{u})=\left[\begin{array}{cc}
0 & 1+\epsilon \\
\alpha^{2} & 0
\end{array}\right]
$$

The eigenvalues are $\lambda_{1,2}(\omega)=\alpha \sqrt{1+\epsilon(\omega)}$. It is clear that the stochastic problem is weakly (strongly) hyperbolic only if $\epsilon \geqslant-1(\epsilon>-1)$. Therefore, while the original deterministic problem is always hyperbolic, the stochastic problem is not whenever the random variable $\epsilon(\omega)$ is not properly bounded, e.g. a Gaussian distribution.

\subsubsection{Governing equations}

Let $\rho_{i}: \Gamma_{i} \rightarrow \mathbb{R}^{+}$be the probability density functions (PDF) of the random variable $y_{i}(\omega), \omega \in \Omega$, and its image $\Gamma_{i} \equiv y_{i}(\Omega) \subset \mathbb{R}$ be intervals in $\mathbb{R}$ for $i=1, \ldots, N$. Then

$$
\rho(Y)=\prod_{i=1}^{N} \rho_{i}\left(y_{i}\right), \quad Y \in \Gamma
$$

is the joint probability density of the random vector $Y=\left(y_{1}, \ldots, y_{N}\right)$ with the support

$$
\Gamma \equiv \prod_{i=1}^{N} \Gamma_{i} \subset \mathbb{R}^{N}
$$

This allows us to conduct numerical formulations in the finite dimensional ( $N$-dimensional) random space $\Gamma$, in replacement of the infinite dimensional space $\Omega$. The stochastic governing equations are: find $\mathbf{v}=[A, U]^{\mathrm{T}}: \mathbb{R} \times[0, T] \times \Gamma \rightarrow \mathbb{R}^{2}$, such that for all $Y \in \Gamma$, the following equation holds:

$$
\frac{\partial \mathbf{v}}{\partial t}(x, t, Y)+\frac{\partial \mathbf{F}(\mathbf{v})}{\partial x}(x, t, Y)=\mathbf{s}(x, t, Y), \quad \text { in } \mathbb{R} \times(0, T] \times \Gamma,
$$

subject to initial condition

$$
\mathbf{v}=\mathbf{g} \quad \text { on } \mathbb{R} \times\{t=0\} \times \Gamma
$$

where $\mathbf{F}=\left(A U, U^{2} / 2+p / \rho\right)^{\mathrm{T}}, \mathbf{s}=(0, f /(\rho A))^{\mathrm{T}}$. In practice, the computational domain is always bounded. It is necessary to replace the unbounded spatial domain $\mathbb{R}$ with a bounded domain $D \in \mathbb{R}$ in the above formulation and supplement with the following boundary condition:

$$
B(\mathbf{v})=\mathbf{0}, \quad \text { on } \partial D \times[0, T] \times \Gamma,
$$

where $B$ is an appropriate boundary condition operator.

In this paper, under the assumption that $y_{i}(\omega)$ are uniformly distributed in $(-1,1)$ for all $i=1, \ldots, N$, we have $\rho_{i}=1 / 2$ and $\Gamma=(-1,1)^{N}$.

\subsection{Generalized polynomial chaos}

To solve system (25)-(27), we employ the generalized polynomial chaos (gPC) expansion.

In $\mathrm{gPC}$ expansion, solutions of the system are expanded as finite-term orthogonal polynomials series in terms of the input random variables, i.e.,

$$
\mathbf{v}_{M}(t, x, Y(\omega))=\sum_{j=1}^{M} \widehat{\mathbf{v}}_{j}(t, x) \Phi_{j}(Y(\omega)),
$$


where $\left\{\Phi_{j}(Y)\right\}_{j=1}^{M}$ are $N$-variate orthogonal polynomials of degree up to $P$. Subsequently, the total number of polynomial basis functions is $M=(N+P) ! /(N ! P !)$. Corresponding to the uniform distribution of $Y$, the orthogonal polynomials $\Phi$ are chosen as Legendre polynomials. For other types of distributions of $Y$, different types of orthogonal polynomials can be chosen to achieve better convergence, e.g., Hermite polynomials for Gaussian distribution. For further details, see [39]. The expansion coefficients can be obtained by

$$
\widehat{\mathbf{v}}_{j}(t, x)=\int_{\Gamma} \mathbf{v}(t, x, Y) \Phi_{j}(Y) \rho(Y) \mathrm{d} Y, \quad j=1, \ldots, M,
$$

where the orthogonal polynomial basis functions have been properly normalized.

\subsubsection{Stochastic collocation method}

The objective is to evaluate the expansion coefficients $\left\{\hat{\mathbf{v}}_{j}\right\}$ using the stochastic collocation method based on sparse grid $[37,36]$. The procedure consists of the following steps:

1. Choose a collocation nodal set (sparse grid) $\left\{Y^{(m)}, \alpha^{(m)}\right\}_{m=1}^{Q}$ in space $(-1,1)^{N}$, where $Y^{(m)}=\left(y_{1}^{(m)}, \ldots, y_{N}^{(m)}\right)$ is the $m$ th-node and $\alpha^{(m)}$ is its corresponding weight;

2. for each $m=1, \ldots, Q$, solve problem (25)-(27) with a fixed parameter set $\beta_{i}^{(m)}=\left\langle\beta_{i}\right\rangle\left(1+\sigma y_{i}^{(m)}\right), i=1, \ldots, N$, via an accurate numerical scheme, to obtain its solution $\widetilde{\mathbf{v}}^{(m)}=\widetilde{A}\left(t, x, Y^{(m)}\right), \widetilde{U}\left(t, x, Y^{(m)}\right)$; (Note this is a deterministic problem.)

3. evaluate the approximate gPC expansion coefficients

$$
\widetilde{\mathbf{v}}_{j}(t, x)=\mathcal{U}^{Q}\left[\widetilde{\mathbf{v}}(Y) \Phi_{j}(Y)\right]=\sum_{m=1}^{Q} \widetilde{\mathbf{v}}^{(m)} \phi_{j}\left(Y^{(m)}\right) \alpha^{(m)}, \quad j=1, \ldots, M
$$

4. and finally, construct the $N$-variate, $P$ th-order gPC approximation

$$
\widetilde{\mathbf{v}}_{M}(t, x, Y)=\sum_{j=1}^{M} \widetilde{\widehat{\mathbf{v}}}_{j}(t, x) \Phi_{j}(Y), \quad M=\left(\begin{array}{c}
N+P \\
N
\end{array}\right) .
$$

The choice of the nodal set $\left\{Y^{(m)}, \alpha^{(m)}\right\}_{m=1}^{Q}$ should be made such that an accurate integration rule can be constructed, i.e., $\mathcal{U}^{Q}[f]=\sum_{m=1}^{Q} f\left(Y^{(m)}\right) \alpha^{(m)} \approx \int_{\Gamma} f(Y) \rho(Y) \mathrm{d} Y$ with sufficient accuracy. By doing so, Eq. (30) is an approximation to the exact coefficients defined by Eq. (29). Subsequently, Eq. (31) is an approximation of the exact gPC expansion (28). It is shown in [36] that the errors of the approximate gPC solution (31) via this stochastic collocation algorithm stem from three sources: $\epsilon_{G}$, finite-term truncation error of gPC expansion (28); $\epsilon_{\Delta}$, deterministic numerical error in solving system (25)-(27); and $\epsilon_{Q}$, error in approximating the integrals in (29) by a discrete integration rule (30). The overall mean-square error can be bounded by

$$
\epsilon \equiv\left(\int_{\Gamma}\left[\mathbf{v}(x, t, Y)-\widetilde{\mathbf{v}}_{M}(t, x, Y)\right]^{2} \rho(Y) \mathrm{d} Y\right)^{1 / 2} \leqslant\left[\epsilon_{G}^{2}+\epsilon_{Q}^{2}+M \epsilon_{\Delta}^{2} C_{Q}^{2}\right]^{1 / 2},
$$

where $C_{Q}$ is a constant associated with the integration rule and independent of all other factors. For details, see [36]. We remark that the major computational efforts will be on the repetitive and deterministic solutions of (25)-(27) for each fixed parameters $Y^{(m)}, m=1, \ldots, Q$. The choice of nodal set is a pre-processing step, and the evaluation of gPC coefficients (30) and reconstruction of the approximate gPC expression (31) are post-processing steps.

Existing studies suggest that for moderately large number of input random variables, the Smolyak sparse grid is an efficient choice [37,36]. The sparse grid is a subset of the full tensor product grids based on onedimensional nodal set, as illustrated in Fig. 2 for a two-dimensional space. It retains the high accuracy resulted from high-order polynomial approximations but employs much less number of nodes in higher dimensional spaces. 

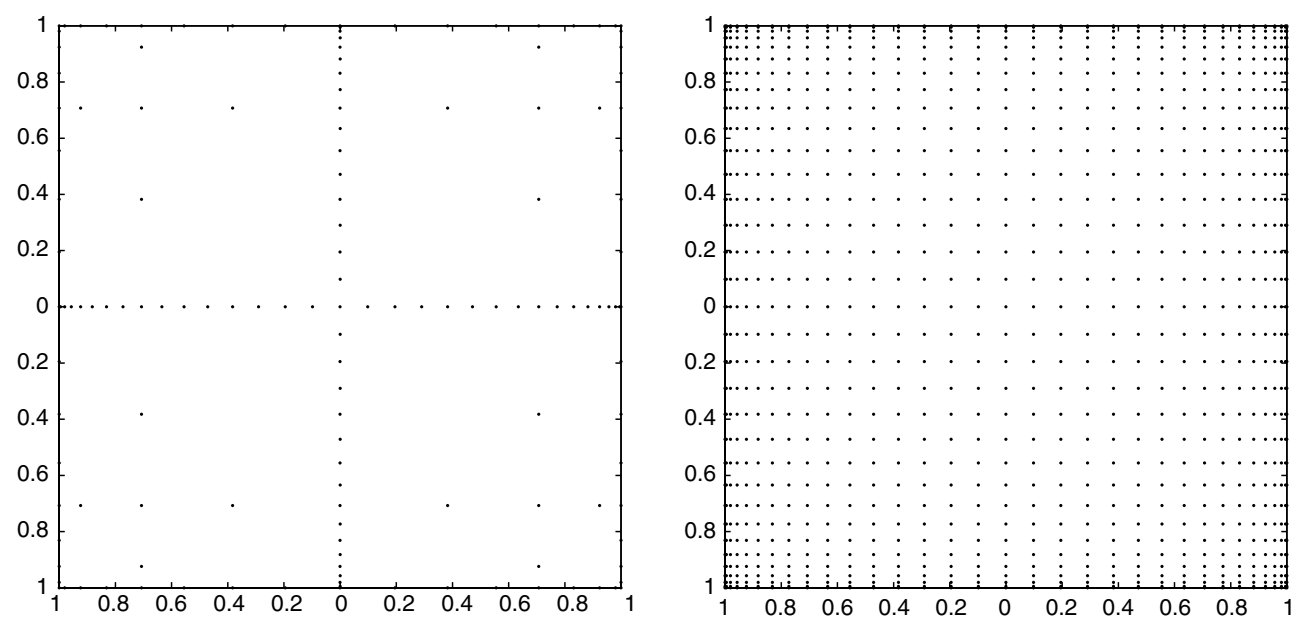

Fig. 2. Two-dimensional $(N=2)$ nodes based on the extrema of Chebyshev polynomials. Left: Sparse grid. Total number of points is 145 . Right: Tensor product grids from the same one-dimensional nodes. Total number of nodes is 1089.

\subsubsection{Solution statistics and sensitivity analysis}

Upon numerical evaluations of all the expansion coefficients via (30), we essentially have an analytical expression of the stochastic solution in a polynomial form of (31), and various post-processing procedures can be carried out to obtain solution statistics. For example, the mean value can be evaluated as

$$
\left\langle\widetilde{\mathbf{v}}_{M}(x, t)\right\rangle \equiv \int \widetilde{\mathbf{v}}_{M}(x, t, Y) \rho(Y) \mathrm{d} Y \approx \mathcal{U}^{Q}\left[\widetilde{\mathbf{v}}_{M}(x, t, Y)\right]=\sum_{m=1}^{Q} \widetilde{\mathbf{v}}^{(m)} \alpha^{(m)} .
$$

Parametric sensitivity analysis can also be conducted in a straightforward manner. Here we define the scaled global sensitivity coefficients with respect to the input random parameters as

$$
S_{\widetilde{\mathbf{v}}, j} \equiv\left\langle\beta_{j}\right\rangle\left\langle\frac{\partial \widetilde{\mathbf{v}}_{M}}{\partial \beta_{j}}\right\rangle=\frac{1}{\sigma} \int \frac{\partial \widetilde{\mathbf{v}}_{M}}{\partial y_{j}} \rho(Y) \mathrm{d} Y, \quad j=1, \ldots, N .
$$

When the numerical gPC approximation (31) is obtained, the above integral can be readily obtained, i.e.,

$$
S_{\widetilde{\mathbf{v}}, j}=\frac{1}{\sigma} \sum_{i=1}^{M} \widetilde{\mathbf{v}}_{i} \int \frac{\Phi_{i}(Y)}{\partial y_{j}} \mathrm{~d} Y
$$

where the integrals of the polynomial derivatives can be evaluated analytically via their definitions.

\section{Numerical results}

To demonstrate the stochastic modelling techniques we consider two examples. In the first test case (Section 3.1) we analyze a small network of three vessels which make a single bifurcation. This example is useful to help motivate the deterministic dynamics and interpret the results from the uncertainty analysis. Then in Section 3.2 we consider a network of 37 arteries compiled to represent the larger arteries of the human systemic system and previous studied in a deterministic manner in $[22,3]$.

\subsection{Case I: One-bifurcation network}

\subsubsection{Deterministic solution}

We start by considering the solution of the deterministic solutions as schematically illustrated in Fig. 3. In this problem we consider three segments of vessel connected to make a single bifurcation. Each segment has a length of $20 \mathrm{~cm}$ and circular cross-section. The parent vessel has a diameter of $1 \mathrm{~cm}$ whilst both the daughter branches have diameters of $1 / \sqrt{6} \mathrm{~cm}$. In the parent vessel the material parameter $\beta$ is set to $\beta=32,497 \mathrm{~g} /$ 
a
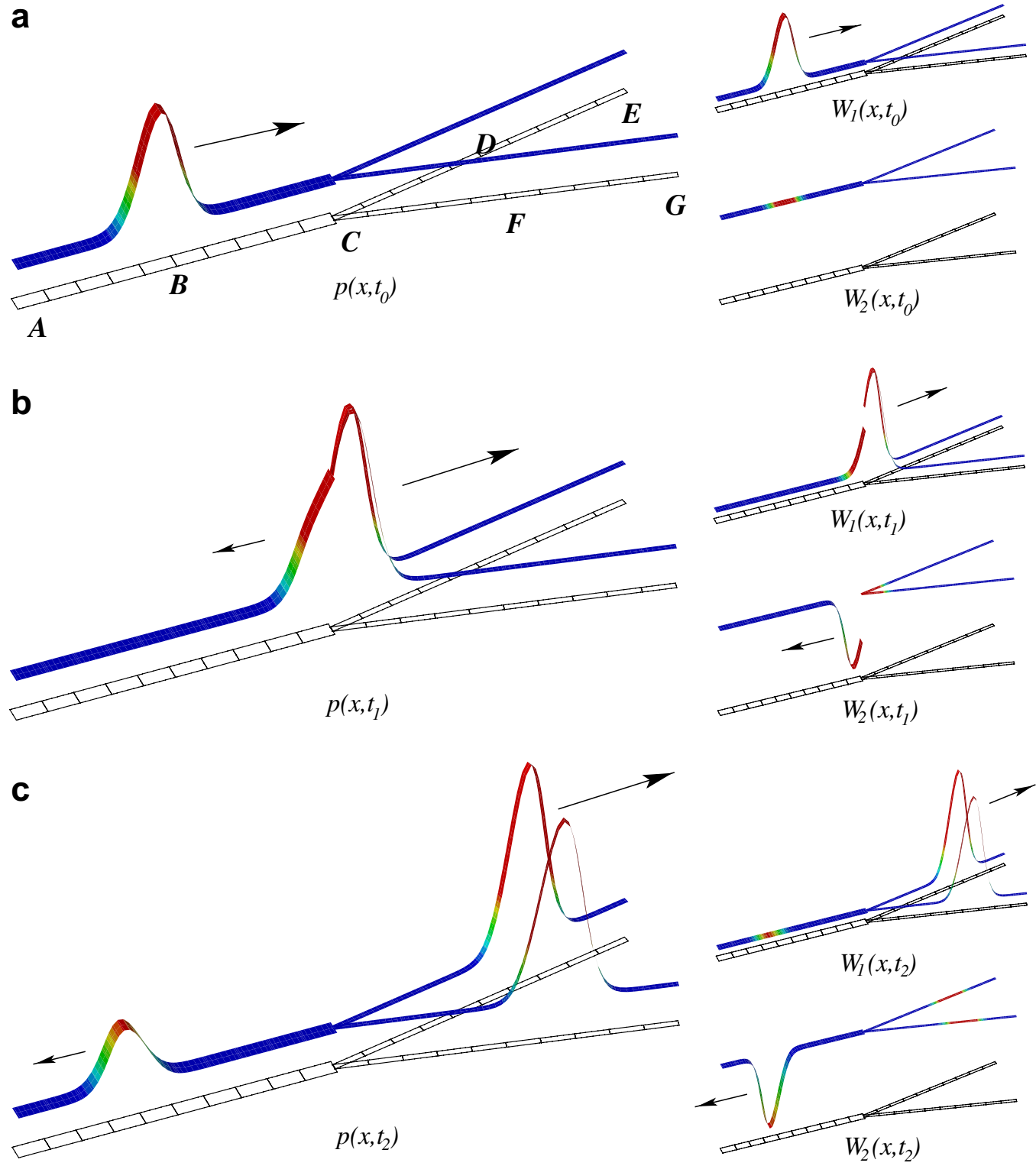

Fig. 3. Computational domain and solution of the pressure wave at time (a) $t_{0}=0.05 \mathrm{~s}$, (b) $t_{1}=0.09 \mathrm{~s}$ and (c) $t_{2}=0.13 \mathrm{~s}$. Also shown as inset plots on the right hand side is the solution in terms of the forward and backward travelling characteristic solutions, $W_{1}(x, t)$ and $W_{2}(x, t)$.

$\left(\mathrm{s}^{2} \mathrm{~cm}^{2}\right)$, whilst in the daughter vessels we have specified that $\beta=79,602 \mathrm{~g} /\left(\mathrm{s}^{2} \mathrm{~cm}^{2}\right)$. Using Eq. (4) we observe that the linearized wave speed, when $\rho=1 \mathrm{~g} / \mathrm{cm}^{3}$, in the parent and daughter vessels are all $c=120 \mathrm{~cm} / \mathrm{s}$. These values are physiologically plausible for a large artery although the wave speed is relatively slow.

For the deterministic discontinuous Galerkin solver, 10 uniform elements are used inside each segment and polynomial order of seven was imposed. A similar discretization was also adopted in all subsequent solution of this problem. History points were placed at the boundaries and in the middle of each domain, labelled as points A-G in the Fig. 3a. As highlighted in Fig. 3, in these tests a relatively short pulse period is imposed at points A through an absorbing velocity condition on the incoming characteristic [26] of the form

$$
U\left(0^{-}, t\right)=U_{\text {peak }} \exp \left(-C\left(t-t_{0}\right)^{2}\right), \quad A\left(0^{-}, t\right)=\frac{\pi}{4},
$$

where $U_{\text {peak }}=1 \mathrm{~cm} / \mathrm{s}, C=5000 \mathrm{~s}^{-2}$ and $t_{0}=0.05 \mathrm{~s}$ is introduced at the inlet of the parent domain (labelled point $\mathrm{A}$ in Fig. 3 (a)). From this definition we observe that the time period over which the pulse velocity 
has a magnitude greater than $1 e-2 \mathrm{~cm} / \mathrm{s}$ is $\Delta t=2 \sqrt{(}-\ln (0.01) /-C) \approx 0.06 \mathrm{~s}$ which is far shorter than a normal heart beat. Further, under a linearized assumption the input pulse wave given by Eq. (35) has a spatial wavelength of approximately $\lambda=c \times \Delta t=7.2 \mathrm{~cm}$. This wavelength is unrealistic since physiological waves typically have wavelengths of the order of $2 \mathrm{~m}$ and longer. However, the use of a short wave allows us to more easily understand the dynamics of the system. The pulse wave solution can also be interpreted as a type of transfer function of the system from which we can deduce the role of longer wavelength input under linear assumptions [33].

The input wave transmits through the parent vessel as shown in Fig. 3a. We observe from the inset plots on the right hand side, which illustrate the solution in terms of the characteristic values, that at this time there are only forward, $W_{1}(x, t)$, travelling waves. As the wave arrives at the bifurcation there is a partial reflection of the pulse back into the parent vessel and an associated transmission of the pulse into the daughter vessels. The reflection leads to the generation of a backward travelling, $W_{2}(x, t)$, pulse in the parent vessel as shown in Fig. $3 \mathrm{~b}$ and $\mathrm{c}$. The size of the reflected pressure perturbation on top of an incoming pressure perturbation can be determined, to first-order, by the linearized reflection coefficient (using the wave speed evaluated at the undeformed area [26]) defined as:

$$
R_{f}=\frac{\frac{A_{1}}{c_{1}}-\frac{A_{2}}{c_{2}}-\frac{A_{3}}{c_{3}}}{\frac{A_{1}}{c_{1}}+\frac{A_{2}}{c_{2}}+\frac{A_{3}}{c_{3}}}=\frac{1}{2},
$$

where the subscript 1 refers to the parent vessel and subscripts 2 and 3 denote the daughter vessels. The transmitted pressure perturbation to the daughter vessel is equivalently defined as $T=1+R_{f}=3 / 2$. Finally, in Fig. 3c we observe that the waves propagate along the various vessels and since in this problem we have directly imposed the boundary conditions on the incoming characteristics there are no reflection and the waves are entirely absorbed at the boundaries.

In the following analysis we will concentrate on the time history of the pressure waves at the history points labelled in Fig. 3a. In Fig. 4 we therefore show the time history for points A-E corresponding to the deterministic simulation outlined in Fig. 3. We observe from the left hand plot of Fig. 4 that the incoming pressure wave generated by Eq. (35) has a peak magnitude of $60 \mathrm{~g} /\left(\mathrm{s}^{2} \mathrm{~cm}\right)$ and the pulse occurs over an approximate time period of $0.063 \mathrm{~s}$. Using a linear analysis, the pulse propagates at $c=120 \mathrm{~cm} / \mathrm{s}$ and hence passes point $\mathrm{B}$, which is located at $x=10 \mathrm{~cm}$ after a time delay of $\Delta t=10 / 120=0.08 \mathrm{~s}$. When the pulse reaches the bifurcation at point $\mathrm{C}$ the linear reflection predicted by Eq. (36) dictates that half of this wave is reflected back into the parent vessel. At point $\mathrm{C}$ we observe the addition of the forward travelling pressure pulse with the reflected wave leading to a pulse of magnitude $3 / 2 \times 60=90 \mathrm{~g} /\left(\mathrm{s}^{2} \mathrm{~cm}\right)$. However, as the reflected wave propagates back,
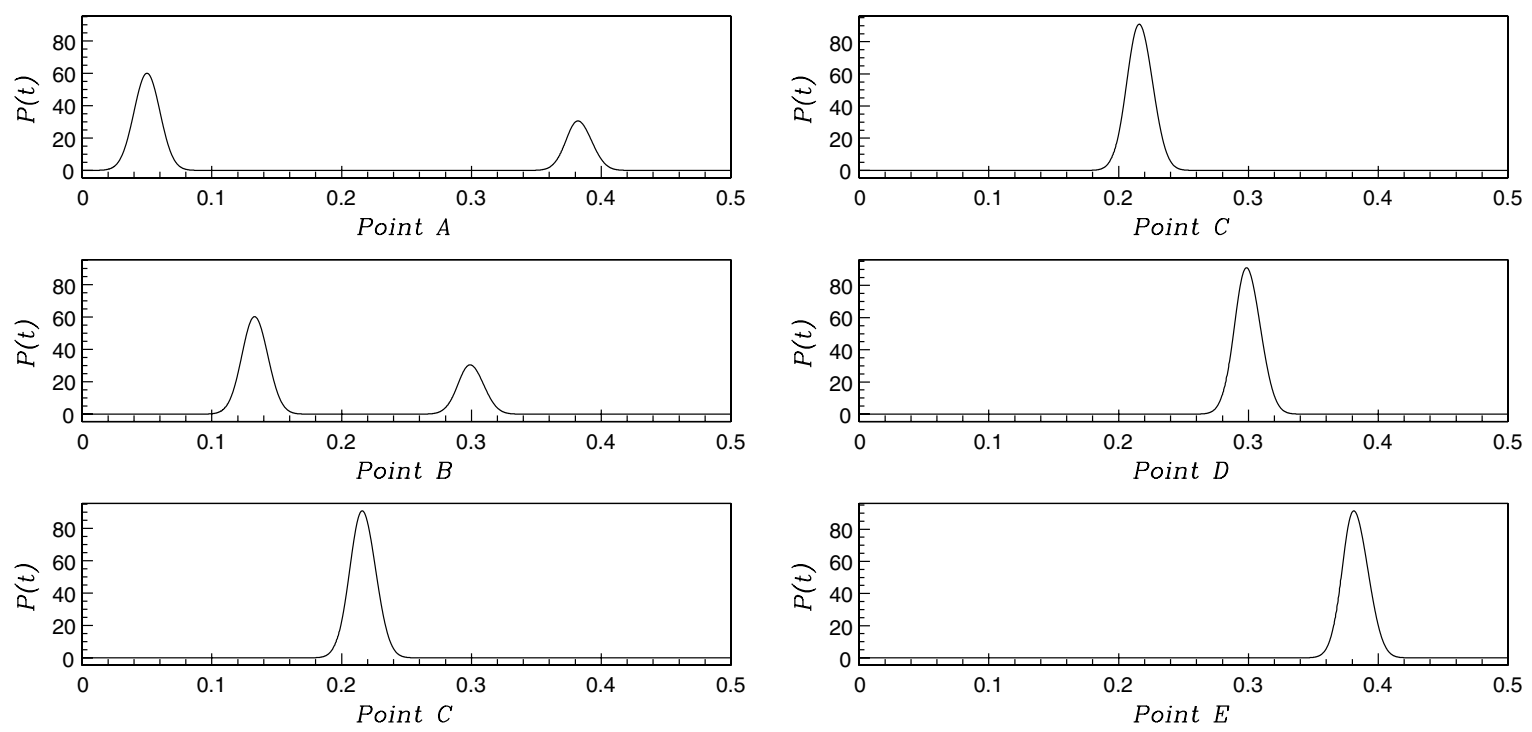

Fig. 4. Time history of pressure $\left(\mathrm{g} /\left(\mathrm{s}^{2} \mathrm{~cm}\right)\right)$ at the history points $\mathrm{A}-\mathrm{E}$ (see Fig. 3a) in domain 1 (parent) and domains 2 and 3 (daughters). Left: domain 1; Right: domains 2 and 3. 
it again passes past points $B$ and then $A$ and has a pulse of magnitude $1 / 2 \times 60=30 \mathrm{~g} /\left(\mathrm{s}^{2} \mathrm{~cm}\right)$. Analogously the transmitted pulse into the daughter vessels has a transmission coefficient of $T=3 / 2$ and so on the right hand plot of Fig. 4 we observe a pulse of magnitude $3 / 2 \times 60=90 \mathrm{~g} /\left(\mathrm{s}^{2} \mathrm{~cm}\right)$ propagating past points D and E also at a speed of $120 \mathrm{~cm} / \mathrm{s}$.

\subsubsection{Stochastic solution: absorbing terminals}

We now consider a $10 \%$ variation in the $\beta$ parameter that depends on the material properties and geometry of each vessel using the uncertainty analysis outlined in Section 2.2. From Eq. (4) we note that there is a square root relationship between $\beta$ and wave speed $c$ and so varying $\beta$ from $0.9 \leqslant \beta /\langle\beta\rangle<1.1$ corresponds to a variation in wave speed of $0.95 \leqslant c /\langle c\rangle \leqslant 1.05$, i.e. a $5 \%$ variation in wave speed. Typically the wave speed (or equivalently the vessel wall characteristics) are difficult to determine either in vivo or in vitro and so this quantity is likely to be a significant source of uncertainty when obtaining data. Once again we prescribe the deterministic inflow conditions given by Eq. (35) in an absorbing characteristic manner and similar to the previous deterministic solutions we use 10 equispaced elements within each vessel with a polynomial expansion of order seven.

Before considering the Stochastic solution we first illustrate the convergence of the solution with respect to the sparse grid of order $k$ in the stochastic collocation method (see [37,36] for more details). With three uncertainty parameters $\beta$ (one for each segment), we have a three-dimensional random space $(N=3)$. For sparse grid collocation method with order $k$, the number of nodes $(Q)$ are $Q=6$ for $k=1 ; Q=25$, for $k=2$; $Q=69$ for $k=3$; and $Q=177$ for $k=4$. In Fig. 5 we therefore plot the time mean and standard deviation at history point A over the time period $0.34 \mathrm{~s}<t<0.42 \mathrm{~s}$ after the incoming pulse wave has reflected at the bifurcation (see also Fig. 4). The convergence with respect to sparse grid order $k$ is also shown in Table 1 where we tabulate the mean and STD root mean square error for each sparse grid order expressed as a percentage of the $k=4$ result.

In Fig. 6 we plot the mean value and standard deviation (STD) of the pressure signal for the history points in the parent vessel and one daughter vessel (i.e. A-E) at $k=4$, similar to the deterministic problem shown in Fig. 4. We have also superimposed the deterministic solution, with a dotted line on top of the mean values. Since the mean represents the ensemble average (with a uniform probability density function) of the pulse wave initially propagating forwards and then reflecting back it is not surprising that the pulse broadens and has a slight decrease in the peak. The broadening physically corresponds to the fact that at points further downstream pulses of faster (or slower) wave-speeds will propagate further (or less) than the deterministic solution. A natural consequence of this observation is also that there is a slight reduction of the peak.

As the pulse wave propagates along each element, the standard deviation increases and exhibits a double peak shape. This form is consistent with an analytical investigation (not shown) involving the linear propagation of a single pulse at varying wave-speeds and then ensemble averaging the solutions (i.e. a Monte Carlo
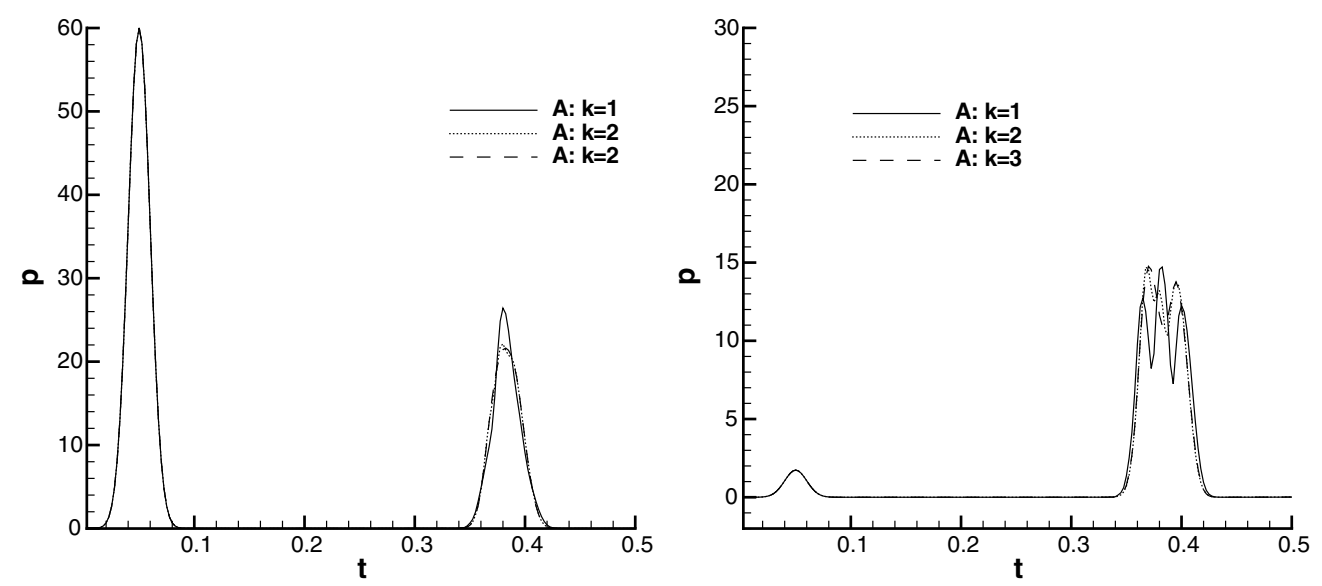

Fig. 5. Time history of the mean (left) and standard deviation (right) of pressure at the history point A in the parent vessel resolved by increasing orders $(k=1,2,3)$ of stochastic methods. Order $k=4$ is indicated by the square symbols. 
Table 1

Relative error as we increase the sparse grid order $k$ with respect to the $k=4$ results

\begin{tabular}{lll}
\hline Stochastic order & Relative error & STD $(\%)$ \\
\cline { 2 - 3 }$k(Q)$ & Mean $(\%)$ & 35 \\
\hline $1(6)$ & 21 & 7.3 \\
$2(25)$ & 3.6 & 0.7 \\
$3(69)$ & 0.3 & \\
\hline
\end{tabular}

Also shown in brackets are the number of nodes $Q$ corresponding to each value of $K$.
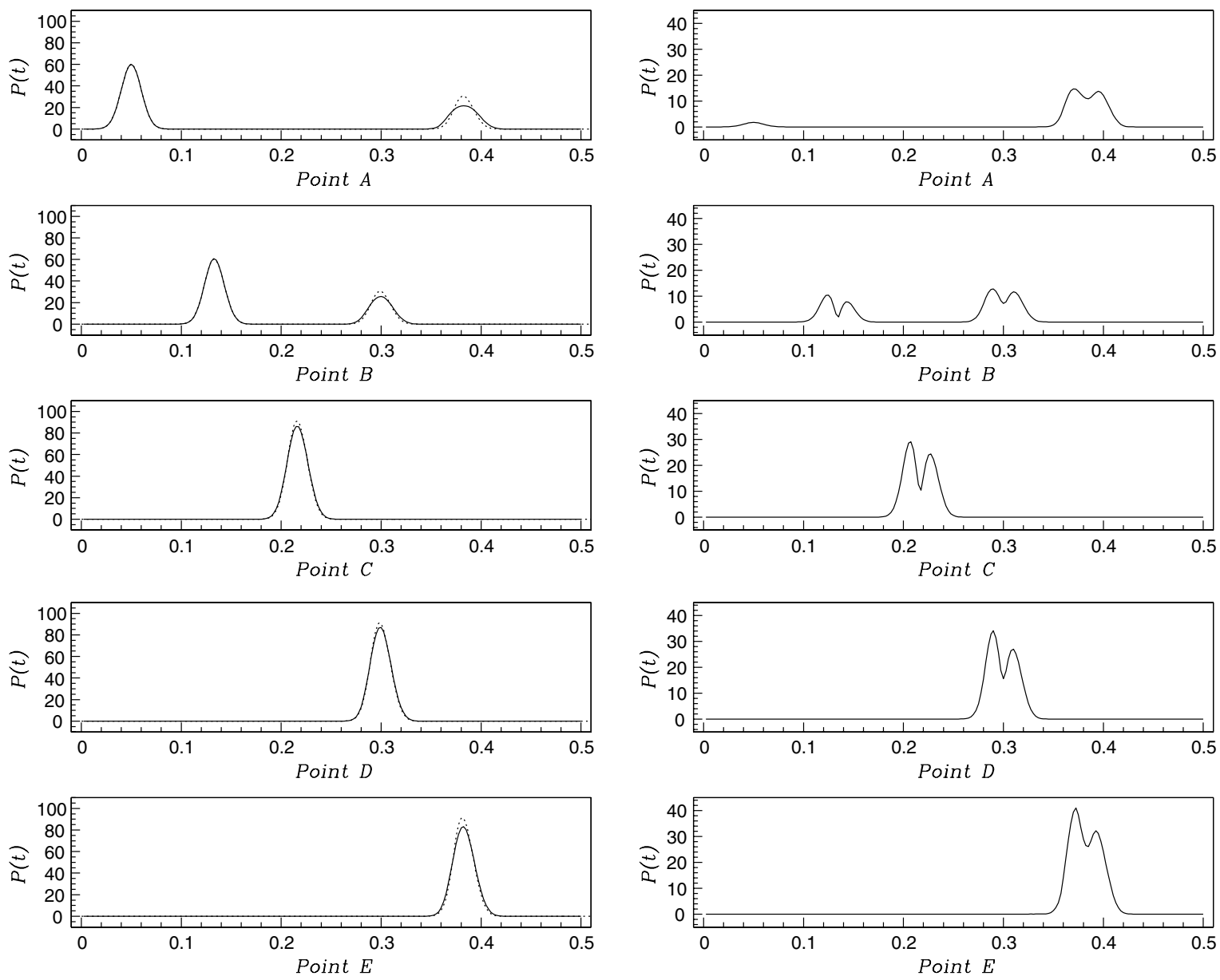

Fig. 6. Time history of the mean (left) and standard deviation (right) of pressure $\left(\mathrm{g} /\left(\mathrm{s}^{2} \mathrm{~cm}\right)\right)$ at the fixed points A-E in domain 1 (Parent) and 2 (daughter). The deterministic solution is superimposed on the mean with a dotted line.

evaluation). However, when we analytically consider the solution of linear pulse wave propagation, the two peaks of the STD distribution are observed to have the same magnitude. We therefore attribute the asymmetry between the two peaks to the non-linearity included in the numerical simulation which leads to a steepening of the compression side of the pulse as it propagates.

We note that a steepening at the front of a pulse wave in space corresponds to a steepening at the back of the pulse wave signal in time. Therefore the higher STD peak in the double peak distribution correlates to the part of the pulse which is spatial steepening. It is also interesting that in Fig. 6 (right) the differential between the STD double peaks is more notable at points B and C when $t<0.25 \mathrm{~s}$. However when the wave reflects (and the steepening of the wave is now on the other side of the pulse) the STD distribution starts to even out. Finally, we notice that at the bifurcation, point $\mathrm{C}$, the standard deviation is significantly higher, which is consistent with the incoming pulse and reflecting wave overlapping at this point. Ignoring the bifurcation point in 
Fig. 6 (right) the increase in the STD occurs at an approximately linear rate of growth in time. The exception being the STD form initial history signal at point A where there is no variation in the wave speed as this inflow condition is directly imposed at this point.

The sensitivity coefficients $S_{P, j}$, defined in Eq. (34), for pressure with respect to the three random input parameters $\beta_{j},(j=1,2,3)$ are evaluated at the history points and shown in Figs. 7 and 8 . We note that $j=1$ is the parent domain and domains 2 and 3 are the daughter vessels. Fig. 7 only shows $S_{P, 2}$ since it is identical to $S_{P, 3}$ due to the symmetry of the problem. The $S_{P, 2}$ signal is roughly two orders of magnitude smaller than $S_{P, 1}$ (note the scales) highlighting that the pressure, in this problem, is only weakly affected by the material properties of the daughter vessels. This low sensitivity is due to the fact there are no reflections from the ends of the daughter vessels. Therefore the only influence on the pressure due to a change in the material properties $\beta_{2,3}$, can be interpreted through the linearized reflection coefficient (Eq. (36)) which will be altered since it is dependent upon the wave speed $c_{j}$ and so is implicitly a function of $\beta_{j}$. We also note that $S_{P, 2}=S_{P, 3}=0$ when the wave is propagating forward along the parent vessel and so the reflection at the bifurcation has not yet entered into the solution. However, once the wave has reflected we observe a small, $S_{P, 2}=S_{P, 3} \approx 1$, contribution to the sensitivity. In addition to the larger magnitude of $S_{P, 1}$ as compared to $S_{P, 2}$ there is also difference in shape of the different sensitivities, since the $S_{P, 2}$ distribution is purely positive in Fig. 7.
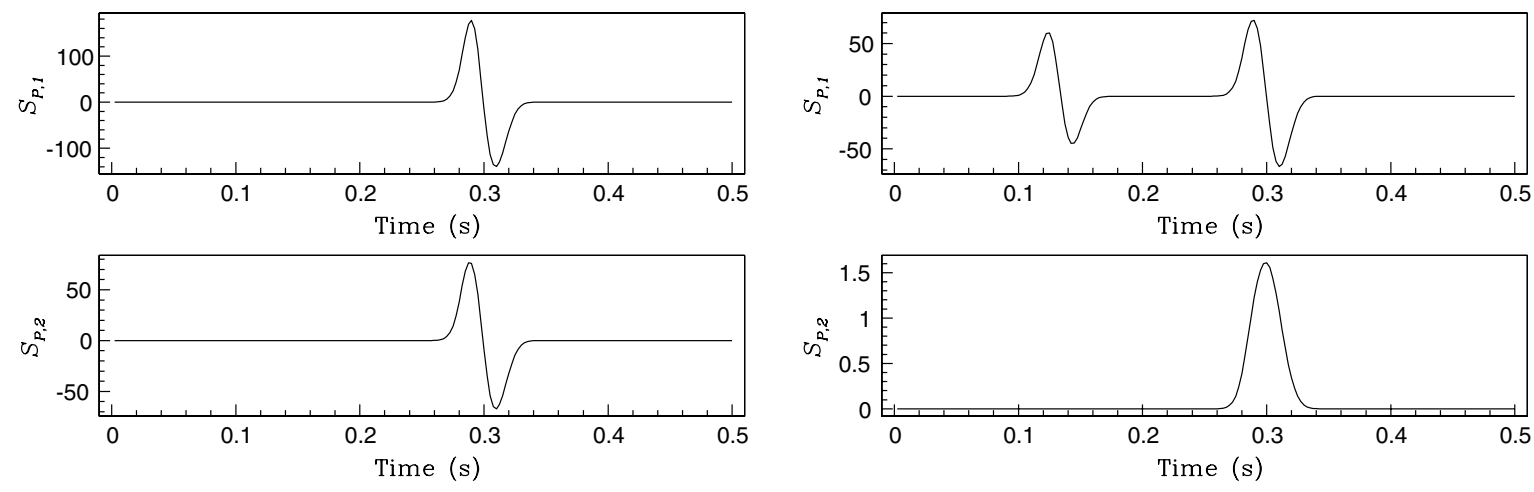

Fig. 7. Sensitivity coefficients of pressure with respect to the random input vessel $\beta_{j}$ in the parent vessel. Left: $S_{P, j}$ at point B; right: $S_{P, j}$ at point $\mathrm{C}, j=1,2,3$.
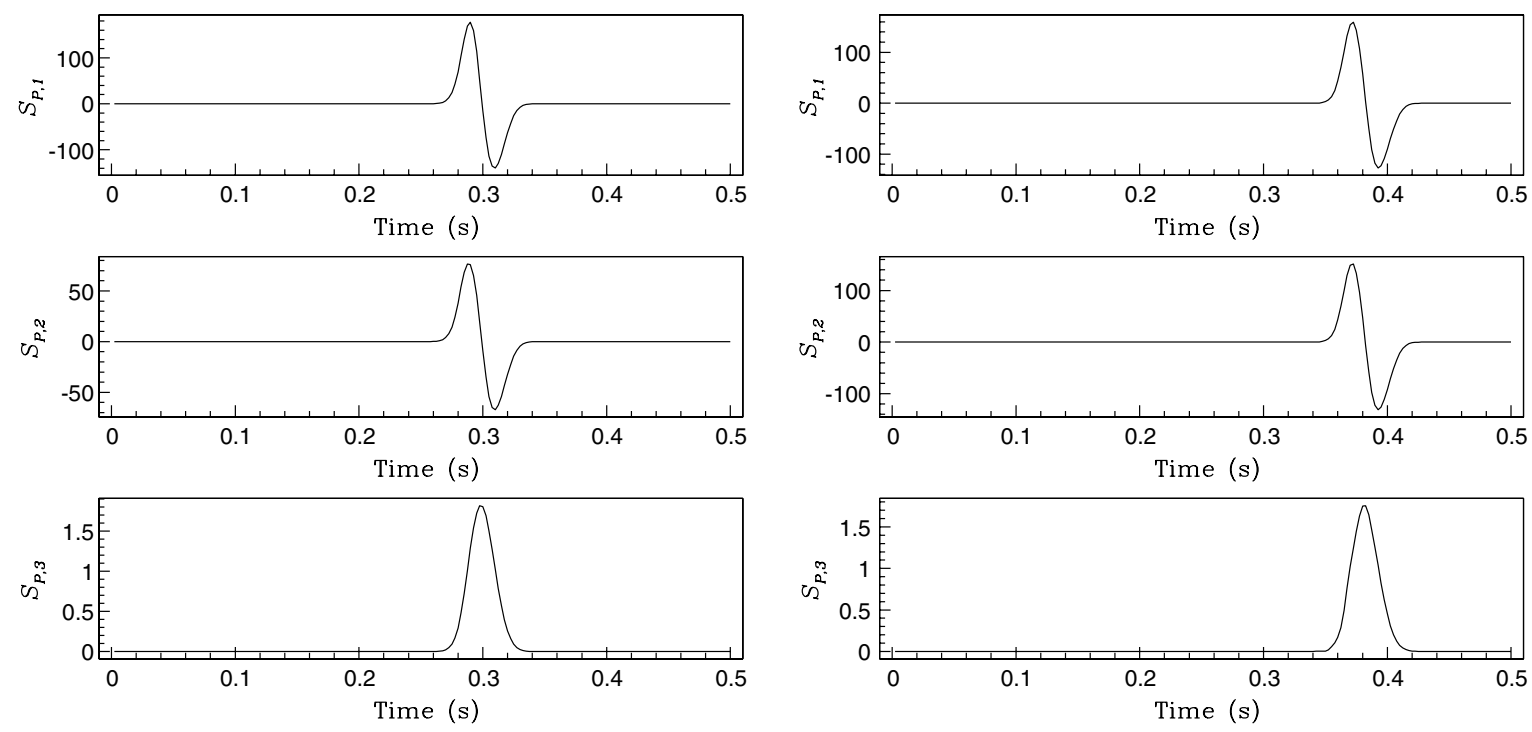

Fig. 8. Sensitivity coefficients of pressure with respect to the random input vessel $\beta_{j}$ in one daughter vessel. Left: $S_{P, j}$ at point D; right: $S_{P, j}$ at point $\mathrm{E}, j=1,2,3$. 
From Eq. (34) we recall that our measure of sensitivity is an ensemble average of $\partial p / \partial \beta_{j}$. Consideration of Eq. (36) leads us to deduce that an increase in $\beta_{2}$ (or $\beta_{3}$ ) will lead to an increased in $c_{2}$ (or $c_{3}$ ) which results in a larger reflection coefficient. Therefore any increase in $\beta_{2}$ or $\beta_{3}$ causes a larger reflected pressure wave and so an increase in $p$ and so results in a positive sensitivity. The shape of $S_{P, 1}$, however, changes sign as the pulse propagates over the history point and this can be attributed to the fact that if we consider a time interval when the pulse is increasing over a history point then an increase in $\beta_{1}$ would lead to an increase in $c_{1}$. The increase in $c_{1}$ results in the pulse being further advanced in time and so has an increased value of pressure (i.e. $\partial P / \partial \beta_{j}>0$ ). Conversely during a time interval where the pulse peak has passed the history point an increase in $\beta_{1}$ (and therefore $c_{1}$ ) would imply a decrease in the pressure value (i.e. $\partial P / \partial \beta_{j}$ ), and hence, cause the sensitivity measure to be negative.

Finally we note that there is a significant increase in the sensitivity at point $\mathrm{C}$ in the parent vessel (note the scale in Fig. 7 (right)). This is consistent with the increase in the mean and standard deviation of the pulse information shown in Fig. 6 and is also consistent with observations of patient monitoring of flow rate signals using ultrasound in coronary arteries [2].

In the daughter vessels, as shown in Fig. 8, the stochastic solutions are directly affected by the upstream incoming wave from the parent vessel (i.e. vary with $S_{P, 1}$ ), as well as the material properties of the local daughter vessel containing the history points (i.e. domain 2 or 3 ). Subsequently the output uncertainty strongly depends on parameters $\beta_{1}$ and $\beta_{2}$ (or $\beta_{3}$ ), and we observe significant sensitivity coefficients of $S_{P, 1}$ and $S_{P, 2}$ with a similar form as $S_{P, 1}$ in the parent vessel. Dependence upon the other daughter vessel (domain 3 ) is again weak and has a similar positive shape for the same reasons as the sensitivity in domain 1 with respect to domains 2 and 3.

\subsubsection{Stochastic solution: terminal reflections}

In more complicated arterial networks we typically expect wave reflections to arise at the end of the terminal vessels. We increase the complexity of the previous example by imposing terminal reflections. In the following example we therefore consider the same one-bifurcation network illustrated in Fig. 3 but now impose a $50 \%$ reflection at the end of the two daughter vessels, i.e. domains 2 and 3. In addition, we strictly impose a reflective pulse velocity at the inflow, rather than on the incoming characteristic, as defined by Eq. (35). Fig. 9 show the mean pressure signal and their standard deviations in the parent and one of the daughter vessels at $k=5$. The explicit enforcement of the velocity condition doubles the peak of the pressure in the previous case shown in Fig. 6. Further, after the initial peak has entered the domain a zero velocity condition is enforced at the inflow which is equal to a $100 \%$ reflection of any backward travelling wave arriving at the inflow at later times.

Fig. 9 shows that up to $t=0.4 \mathrm{~s}$ the history points are analogous to the previous case shown in Fig. 4 . We recall these initial signals correspond to the pulse propagating down the parent vessel and then a $50 \%$ reflection occurs at the bifurcation. At history point $\mathrm{A}$ when $t \approx 0.4 \mathrm{~s}$ we observe that the reflected pulse has a higher peak than when it passed point $\mathrm{B}$ at $t \approx 0.3 \mathrm{~s}$ because at this later time it is now reflected at the inflow. The increase in the peak is therefore analogous to the increase in pulse peak at the pulse hits the bifurcation (i.e. point $\mathrm{C}$ at $t \approx 0.2 \mathrm{~s}$ ). In this reflecting case we now observe at history point $\mathrm{B}$ at $t \approx 0.47 \mathrm{~s}$ that the reflected wave from the inflow is again propagating down the parent vessel. At the same time $t \approx 0.47 \mathrm{~s}$ at history point $\mathrm{D}$ we observe that the initial pulse wave has transmitted to the daughter vessels and $50 \%$ of this pulse has been reflected back towards the bifurcation. When the backwards travelling pulses in the daughter vessels reach the bifurcation, application of the equivalent to Eq. (36) for a wave entering from the daughter vessel tells us that the pressure wave will reflect with a coefficient $R_{f}=-3 / 4$ and will transmit a wave of $1 / 4$ magnitude back into the parent vessel. Due to the symmetry of this model, there is also a pulse arriving at the bifurcation from the parent vessel (of similar magnitude) which is transmitted to the daughter vessels with a multiplying factor of $3 / 2$. The reflected pulse in the daughter vessel therefore has a magnitude of $(3 / 2-3 /$ 4) $\times 1 / 2=3 / 8$ and this pulse of reduced magnitude is evident at time $t \approx 0.65 \mathrm{~s}$, in point $\mathrm{D}$ (Fig. 9).

Figs. 10 and 11 shows the sensitivity coefficient of pressure (see Eq. (34)) in the parent (domain 1) and daughter (domains 2 and 3) vessels with respect to the three input random variables $\beta_{j}, j=1,2$, 3. In Fig. 10 we see the time history at points A and B. We have only plotted the sensitivity with respect to one daughter vessel since the symmetry of the problem means that $S_{P, 2}=S_{P, 3}$. In contrast to Fig. 7 we observe 

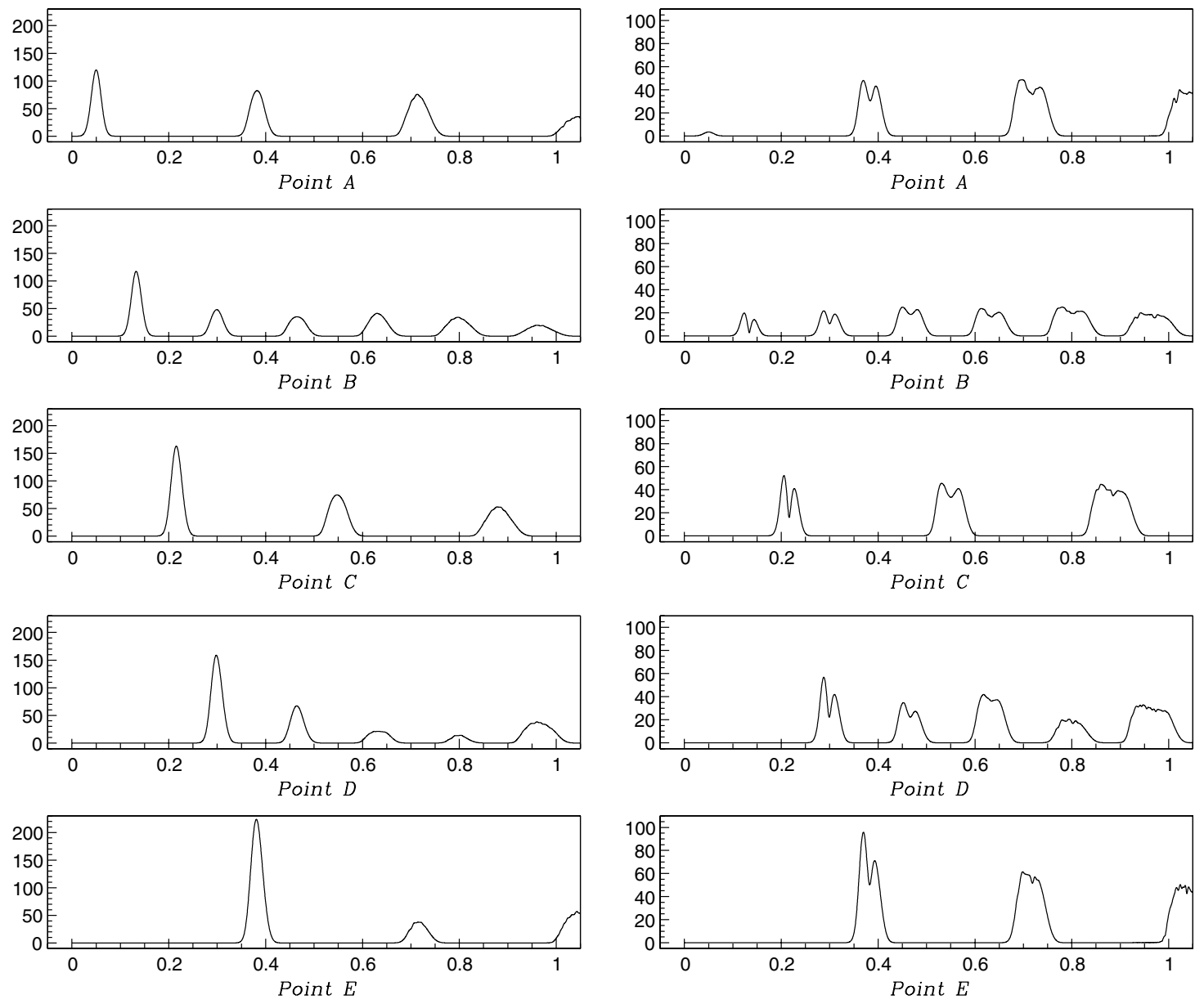

Fig. 9. Time history of pressure $\left(\mathrm{g} /\left(\mathrm{s}^{2} \mathrm{~cm}\right)\right)$ at history points A-E in the parent and a daughter domain for the reflecting termination model. Left: mean; Right: standard deviation.
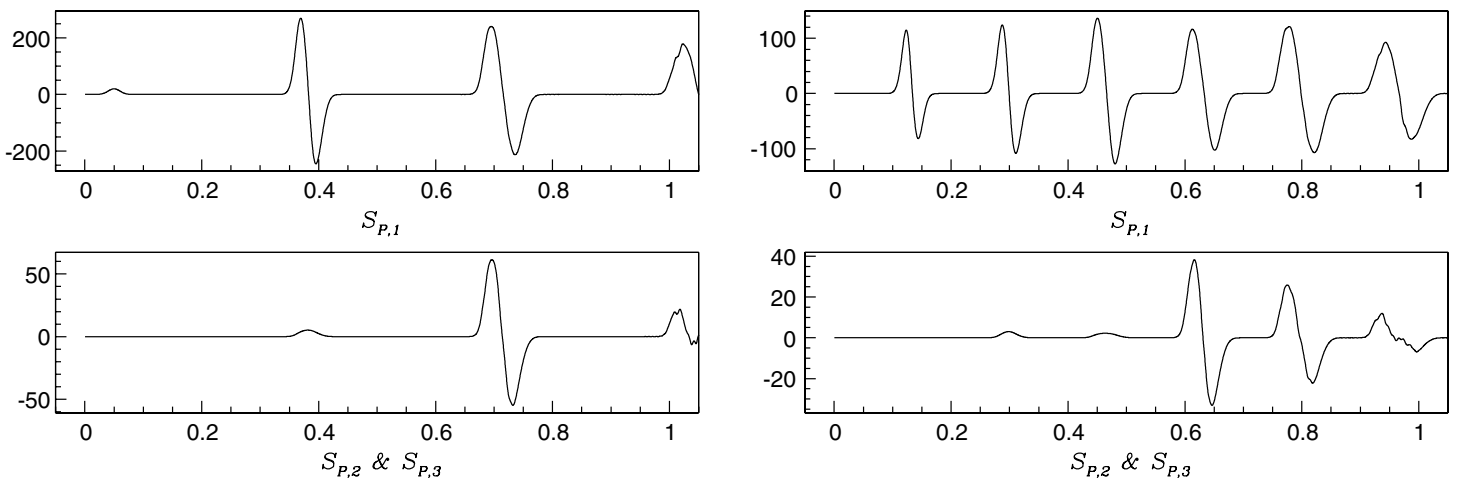

Fig. 10. Time history of sensitivity $S_{P, j}\left(\mathrm{~g} /\left(\mathrm{s}^{2} \mathrm{~cm}\right)\right), j=1,2$ in the parent domain at points A (left) and B (right).

that the history points in the parent vessel now have a notable sensitivity to the $\beta$ parameter in the daughter vessels. Up to time $t \approx 0.4$ the system is analogous to the previous example and the sensitivity $S_{P, 2}=S_{P, 3}$ is still small compared to $S_{P, 1}$. However, by $t \approx 0.7 \mathrm{~s}$ we observe a sensitivity in $S_{P, 2}$ and $S_{P, 3}$ which is approximately $25 \%$ the magnitude of $S_{P, 1}$. At this time a wave has propagated through the daughter vessel, reflected at the domain termination and travelled back to the inflow.

Fig. 11 shows the time history sensitivity at points D and E. Overall we observe similar dynamics to the points in the parent vessel (Fig. 10) and the sensitivities are of equivalent magnitude to Fig. 8. However 

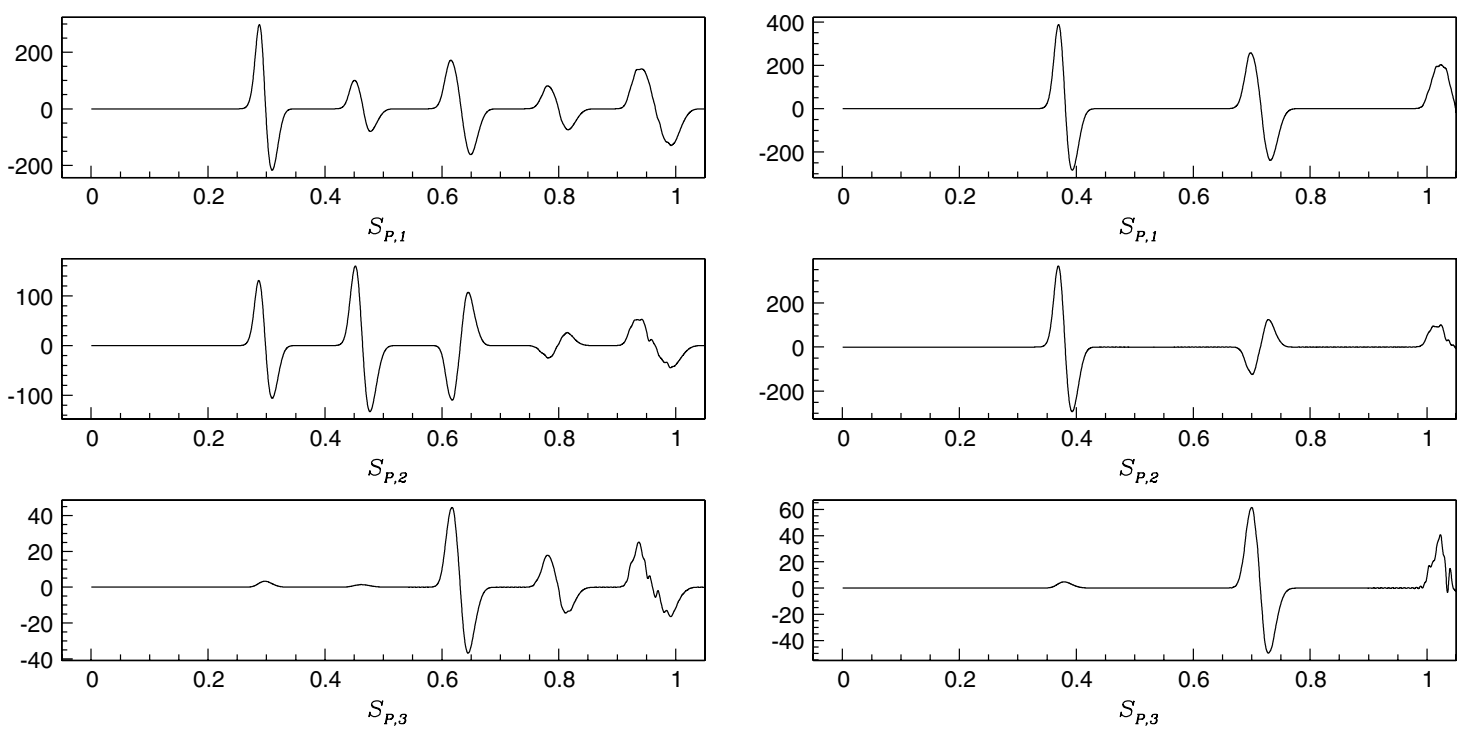

Fig. 11. Time history of sensitivity $S_{P, j}\left(\mathrm{~g} /\left(\mathrm{s}^{2} \mathrm{~cm}\right)\right), j=1,2,3$ in the daughter domain at points $\mathrm{D}$ (left) and $\mathrm{E}$ (right).

one new pattern which can be identified is in the $S_{P, 2}$ history at $t \approx 0.65 \mathrm{~s}$ where the shape of the sensitivity distribution is reversed when compared to other pulse wave passing history points. We recall that at this point a pulse has propagated down the daughter vessel, reflected at the termination of the system and then finally reflected at the bifurcation. This last reflection has a negative coefficient, $R_{f}=-3 / 4$ and therefore the sensitivity, $S_{P, 2}$ with respect to the daughter vessel is reversed.

\subsection{Case II: Realistic network of 37 vessels}

To present a more applied application we consider the case of a network of 37 vessels connected via 16 bifurcations as shown in Fig. 12a and for which geometric information is provided in Table 2. This network is taken from a in vitro model of the larger arteries in the human. A comparison of the deterministic non-linear, one-dimensional model with in vitro data has been previously undertaken and reported in [22]. In this
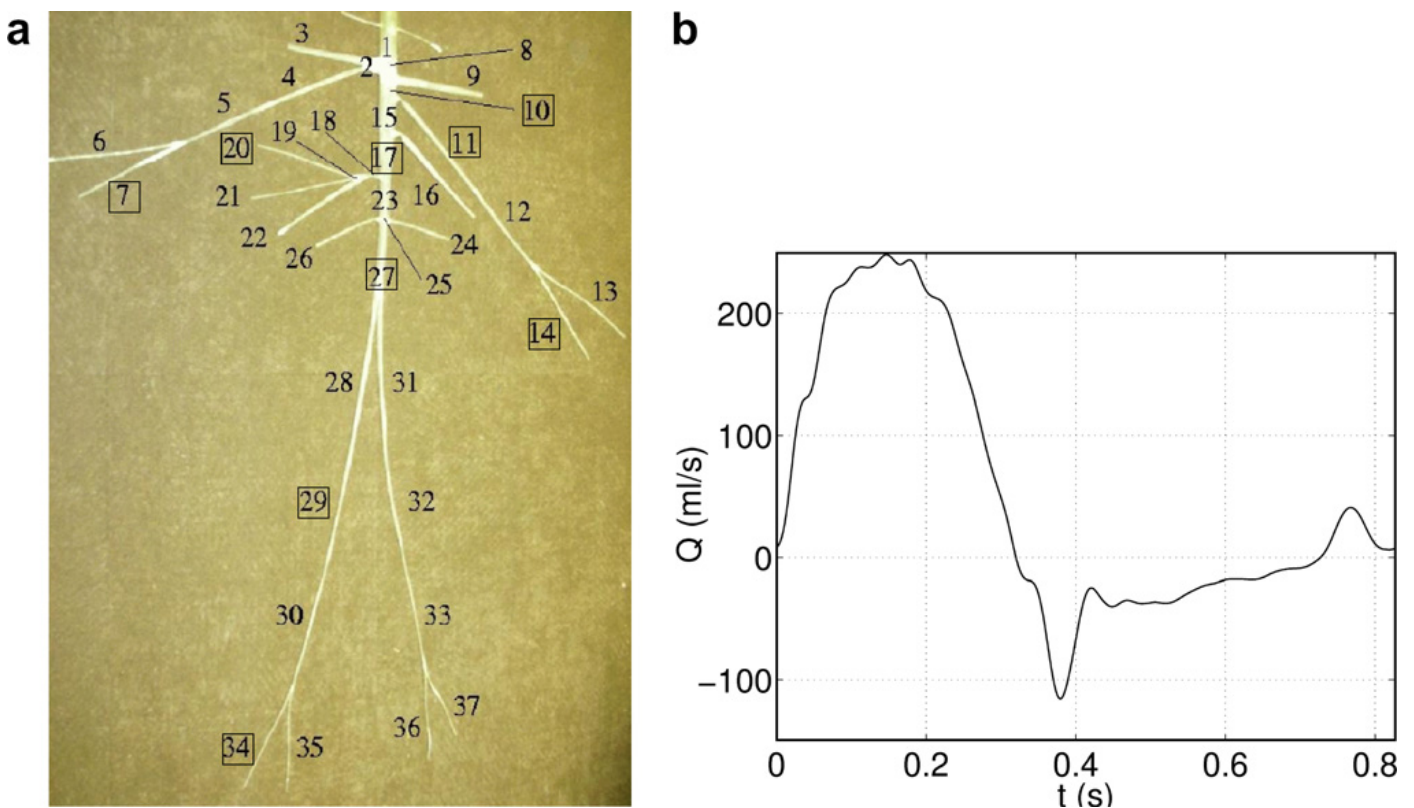

Fig. 12. (a) Thirty-seven vessel in vitro network. (b) The experimental flow rate measured at the ascending aorta enforced as the inflow boundary condition. 
Table 2

Data of the 37 silicon tubes shown in Fig. 12

\begin{tabular}{|c|c|c|c|c|c|}
\hline Arterial segment & $l(\mathrm{~mm}) \pm 2.0 \%$ & $r_{\text {in }} \rightarrow r_{\text {out }}(\mathrm{mm}) \pm 3.5 \%$ & $h(\mathrm{~mm}) \pm 2.5 \%$ & $c_{\text {in }} \rightarrow c_{\text {out }}(\mathrm{m} / \mathrm{s})$ & $R_{\mathrm{p}}\left(10^{9} \mathrm{~Pa} \mathrm{~s} \mathrm{~m}^{-3}\right)$ \\
\hline 1. Ascending aorta & 36 & $14.40 \rightarrow 13.00$ & 0.51 & $5.21 \rightarrow 5.49$ & - \\
\hline 2. Innominate & 28 & $11.00 \rightarrow 7.29$ & 0.35 & $4.89 \rightarrow 6.01$ & - \\
\hline 3. R. carotid & 145 & $5.37 \rightarrow 3.86$ & 0.28 & $6.35 \rightarrow 7.49$ & 2.67 \\
\hline 4. R. subclavian I & 218 & $4.36 \rightarrow 3.34$ & 0.27 & $6.87 \rightarrow 7.84$ & - \\
\hline 5. R. subclavian II & 165 & $3.34 \rightarrow 2.78$ & 0.16 & $6.00 \rightarrow 6.58$ & - \\
\hline 6. R. radial & 235 & 2.07 & 0.15 & 7.43 & 3.92 \\
\hline 7. R. ulnar & 177 & 2.10 & 0.21 & 8.81 & 3.24 \\
\hline 8. Aortic arch I & 21 & $13.00 \rightarrow 12.50$ & 0.50 & $5.41 \rightarrow 5.52$ & - \\
\hline 9. L. carotid & 178 & $5.58 \rightarrow 3.73$ & 0.31 & $6.55 \rightarrow 8.00$ & 3.11 \\
\hline 10. Aortic arch II & 29 & $12.50 \rightarrow 11.80$ & 0.41 & $4.98 \rightarrow 5.12$ & - \\
\hline 11. L. subclavian I & 227 & $4.42 \rightarrow 3.39$ & 0.22 & $6.21 \rightarrow 7.10$ & - \\
\hline 12. L. subclavian II & 175 & $3.39 \rightarrow 2.84$ & 0.17 & $6.26 \rightarrow 6.84$ & - \\
\hline 13. L. radial & 245 & 2.07 & 0.21 & 8.84 & 3.74 \\
\hline 14. L. ulnar & 191 & 2.07 & 0.16 & 7.77 & 3.77 \\
\hline 15. Thoracic aorta I & 56 & $11.80 \rightarrow 11.00$ & 0.43 & $5.29 \rightarrow 5.48$ & - \\
\hline 16. Intercostals & 195 & $4.12 \rightarrow 3.22$ & 0.27 & $7.07 \rightarrow 7.99$ & 2.59 \\
\hline 17. Thoracic aorta II & 72 & $11.00 \rightarrow 9.26$ & 0.34 & $4.84 \rightarrow 5.26$ & - \\
\hline 18. Celiac I & 38 & 3.97 & 0.20 & 6.20 & - \\
\hline 19. Celiac II & 13 & 4.31 & 1.25 & 14.90 & - \\
\hline 20. Splenic & 191 & 1.83 & 0.13 & 7.24 & 3.54 \\
\hline 21. Gastric & 198 & 1.92 & 0.11 & 6.73 & 4.24 \\
\hline 22. Hepatic & 186 & $3.31 \rightarrow 2.89$ & 0.21 & $6.95 \rightarrow 7.44$ & 3.75 \\
\hline 23. Abdominal aorta I & 62 & $9.26 \rightarrow 8.01$ & 0.33 & $5.19 \rightarrow 5.59$ & - \\
\hline 24. L. renal & 120 & 2.59 & 0.19 & 7.39 & 3.46 \\
\hline 25. Abdominal aorta II & 7 & 7.90 & 0.35 & 5.83 & - \\
\hline 26. R. renal & 118 & 2.55 & 0.16 & 6.95 & 3.45 \\
\hline 27. Abdominal aorta III & 104 & $7.80 \rightarrow 5.88$ & 0.30 & $5.41 \rightarrow 6.24$ & - \\
\hline 28. R. iliac-femoral I & 205 & $3.9 \rightarrow 3.38$ & 0.21 & $6.47 \rightarrow 6.94$ & - \\
\hline 29. R. iliac-femoral II & 216 & $3.38 \rightarrow 2.31$ & 0.15 & $5.89 \rightarrow 7.13$ & - \\
\hline 30. R. iliac-femoral III & 206 & $2.31 \rightarrow 2.10$ & 0.20 & $8.04 \rightarrow 8.44$ & - \\
\hline 31. L. iliac-femoral I & 201 & $4.02 \rightarrow 3.34$ & 0.20 & $6.19 \rightarrow 6.79$ & - \\
\hline 32. L. iliac-femoral II & 195 & $3.34 \rightarrow 2.26$ & 0.16 & $6.11 \rightarrow 7.44$ & - \\
\hline 33. L. iliac-femoral III & 207 & $2.26 \rightarrow 2.12$ & 0.13 & $6.67 \rightarrow 6.89$ & - \\
\hline 34. R. anterior tibial & 163 & 1.55 & 0.15 & 8.47 & 5.16 \\
\hline 35. R. posterior tibial & 151 & 1.53 & 0.12 & 7.73 & 5.65 \\
\hline 36. L. posterior tibial & 149 & 1.58 & 0.11 & 7.23 & 4.59 \\
\hline 37. L. anterior tibial & 126 & 1.55 & 0.10 & 7.01 & 3.16 \\
\hline
\end{tabular}

$l$, length; $r_{\text {in }} \rightarrow r_{\text {out }}$, diastolic cross-sectional radii at the inlet and the outlet, linearly tapered in between; $h$, average wall thickness; $c_{\text {in }} \rightarrow c_{\text {out }}$, wave speed at the inlet and the outlet; $R_{\mathrm{p}}$, peripheral resistance. Single numbers indicate vessels with a constant cross-section. The interval of confidence of the geometrical measurements is indicated in the heading. Data taken from [22].

study the outflow boundary conditions were treated in the most straightforward, reproducable experimental manner by attaching a rigid tube to the outflow segment which were then attached to overflow reservoirs of fixed height. This type of terminal boundary condition does not give physiological reflections because peripheral compliance is not simulated. However, the experimental uncertainty is minimized by such an approach which was the objective of the study in [22].

The rigid tube attached to the outlet of each terminal branch, together with the overflow reservoir at constant height, were simulated as outflow boundary conditions by enforcing the relation

$$
Q_{1 \mathrm{D}}=\frac{p_{1 \mathrm{D}}-p_{\mathrm{out}}}{R_{\mathrm{p}}}
$$

where $Q_{1 \mathrm{D}}$ and $p_{1 \mathrm{D}}$ are the flow rate and pressure at the outlet of the one-dimensional terminal branch, $p_{\text {out }}=3.2 \mathrm{mmHg}$ is the constant hydrostatic pressure measured at the overflow reservoir, and $R_{\mathrm{p}}$ is the peripheral resistance to flow, determined from mean pressure and flow measurements close to the outlet of each terminal branch and listed in Table 2. 
As in our previous examples, we study the effect of introducing a $10 \%$ uncertainty into the elastic parameter $\beta(x)$. The size of the network requires a larger stochastic space-the introduction of 37 independent random variables in each vessel results in a 37-dimensional $(N=37)$ random space. The simulations were performed with a stochastic order of $k=2$ and even at this relatively lower resolution, $Q=2813$ independent "sampling" simulations are required. Although this is a lower stochastic order than those used in our previous problems, the temporal wavelength of this more physiological problem is much larger which reduces the complexity of the solution in the stochastic space. Thus, similar to the non-reflective model considered in Section 3.1.2, $k=2$ should produce reasonably accurate statistics for at least the first two moments (mean and variance). Moreover, existing numerical evidence indicates that for many problems, both time independent and time dependent, the sparse grid stochastic collocation method with $k=2$ delivers comparable, if not more, accurate results than Monte Carlo simulations with at least 10,000 realizations (see [37] for comparisons in random dimensions as high as 50). Following the approach in [22] we consider a series of nine representative vessels taken from the main aorta and within the first, second and third generations of the bifurcating network. These vessels are highlighted with a square in Fig. 12(left). Within the top level vessels we consider the aortic arch II (vessel 10), thoracic aorta II (vessel 17) and abdominal aorta III (vessel 27). Within the first generation we consider the left subclavian I (vessel 11) and right iliac-femoral II (vessel 29), within the second generation of vessels we consider the left ulnar (vessel 14) and right anterior tibial (vessel 34), and within the third generation the right ulnar (vessel 7) and splenic (vessel 20).

The simulations were run over a time interval of $10 \mathrm{~s}$ which allowed the network to reach a time periodic state. Table 3 shows the maximum and minimum value of the pressure sensitivity $S_{P, j}$ with respect to the subset of vessels considered. The sensitivity was evaluated at the centre of each vessel and the maximum and minimum were taken over the last six cycles of this simulation. Each row of the table corresponds to a single vessel and therefore each entry in a row denotes the maximum and minimum sensitivity of this vessel with respect to changes in the elastic properties of the other vessels.

To help interpret the data in Table 3 we can consider some physical properties of the arterial network. Under normal physiological conditions, we expect that forward travelling waves from the aorta are wellmatched at each bifurcation. This necessarily implies that reflected waves are not well-matched when propagating toward the root of the arterial tree and this leads to a wave trapping phenomena, in which backward travelling waves are trapped in the lower generations of the tree. This is consistent with the observation in [22] that pulse waveforms in the aorta have a physiological shape, which means that they are not significantly influenced by the reflective terminal boundaries due to wave trapping phenomena.

Table 3

Minimum and maximum values of the pressure sensitivity $S_{P, j}$ evaluated over the last six cycles with respect to a selection of representative vessels in the 37 network

\begin{tabular}{|c|c|c|c|c|c|c|c|c|c|c|c|c|c|c|c|c|c|c|}
\hline \multirow{4}{*}{$\begin{array}{l}\text { Vessel no. } \\
\\
10\end{array}$} & \multicolumn{18}{|c|}{ Min. and max. pressure sensitivity $S_{P, j}$ with respect to vessel } \\
\hline & \multicolumn{6}{|l|}{ Aorta } & \multicolumn{4}{|c|}{ First generations } & \multicolumn{4}{|c|}{ Second generations } & \multicolumn{4}{|c|}{ Third generations } \\
\hline & 10 & & 17 & & 27 & & 11 & & 29 & & 14 & & 34 & & 7 & & 20 & \\
\hline & -75 & 84 & -176 & 176 & -110 & 167 & -48 & 52 & -90 & 137 & -20 & 21 & -53 & 80 & -15 & 14 & -36 & 30 \\
\hline 17 & -75 & 97 & -148 & 193 & -117 & 218 & -54 & 58 & -69 & 152 & -22 & 20 & -29 & 70 & -16 & 17 & -31 & 27 \\
\hline 27 & -104 & 114 & -198 & 254 & -294 & 367 & -59 & 61 & -266 & 385 & -26 & 22 & -104 & 184 & -17 & 21 & -81 & 72 \\
\hline 11 & -96 & 96 & -234 & 238 & -179 & 156 & -589 & 550 & -117 & 108 & -210 & 196 & -44 & 87 & -29 & 36 & -50 & 44 \\
\hline 29 & -135 & 162 & -372 & 496 & -485 & 654 & -117 & 130 & -871 & 928 & -52 & 53 & -425 & 483 & -39 & 35 & -106 & 92 \\
\hline 14 & -108 & 120 & -281 & 274 & -230 & 254 & -530 & 538 & -183 & 227 & -674 & 581 & -113 & 138 & -44 & 49 & -53 & 46 \\
\hline 34 & -235 & 288 & -469 & 527 & -605 & 722 & -105 & 143 & -967 & 1315 & -93 & 84 & -698 & 1192 & -61 & 62 & -142 & 129 \\
\hline 7 & -142 & 122 & -244 & 278 & -201 & 227 & -119 & 119 & -188 & 202 & -79 & 81 & -86 & 124 & -351 & 371 & -49 & 40 \\
\hline 20 & -118 & 147 & -246 & 232 & -376 & 394 & -68 & 90 & -286 & 287 & -29 & 43 & -119 & 100 & -15 & 19 & -534 & 618 \\
\hline
\end{tabular}

Each row of the table represents a single vessel and the entries within that row represent the sensitivity with respect to the variation of the elastic parameter $\beta$ in another vessel. 
Taking the wave trapping characteristic of the network into consideration we would expect that rows of the table corresponding to the uppermost vessels are relatively insensitive to changes in lower generations. Considering the first three rows of Table 3 we observe that the sensitivity on average decreases with each generation. Analogously, the rows corresponding to lower generations are significantly affected by the generations higher up the tree but are relatively insensitive to lower generations. This means that the entries beneath the diagonal of the table has a higher sensitivity than those above the diagonal.

The diagonal block components of the table demonstrate a reasonably high sensitivity due to their local influence. However, we observe that the diagonal block are not necessarily symmetric. This reflects the fact that the transmission of waves, for example, from vessel 14 to 34 , is not identical to the transmission of waves from 34 to 14 . Within each diagonal block we note that the smaller vessels tend to have a higher sensitivity than the larger vessels. This is due to the fact that the smaller vessels have a higher wave speed due to smaller diameters and stiffer material properties.

The largest variation in the data is between vessel 34 due to variations in vessel 29. We see from Fig. 12 that vessel 29 is part of the long femoral vessel feeding into the terminal vessel 34 . Therefore, variation in the material properties and wave speed allows the potential for a large phase difference, and therefore large sensitivities, in the signals within vessel 34 .

\section{Conclusions}

We present a technique to carry out a systematical study of the effects of parametric uncertainty in pulse wave propagation in reduced models of human arterial networks. This technique can be applied to any hyperbolic system of equations with random parameters bounded to maintain the hyperbolic nature of the problem. High-order numerical schemes were used in both random space and physical space - a sparse grid stochastic collocation method based on generalized polynomial chaos and a discontinuous Galerkin method with a spectral/ $h p$ discretization, respectively. Detailed numerical simulations were first conducted in an arterial network with one-bifurcation and reflective and absorbing boundary conditions, where we show and study the effects of uncertainty in wave patterns and sensitivity. We then apply the numerical technique to a network of the largest arteries in the human. We have shown that the sparse grid stochastic collocation method [36,37] provides a feasible method for accurate uncertainty analysis of such a random hyperbolic system with large dimensions of random inputs (37-dimensional random space). The sensitivity of pressure in each tree segment with respect to the material and geometrical uncertainty in the $\beta(x)$ parameter was analyzed in each example and we are able to quantify the degree of sensitiveness of pressure distribution induced by each uncertain inputs. Such results present a first systematical attempt in analyzing uncertainty in a human artery tree.

\section{Acknowledgments}

The authors acknowledge Dr. Jordi Alastruey for his helpful comments on the paper and providing the data for the 37 arterial network. We thank Professor George Karniadakis for helping to promote the research behind this paper. The second author acknowledge support from an EPSRC Advanced Research Fellowship.

\section{References}

[1] S. Acharjee, N. Zabaras, Uncertainty propagation in finite deformations-a spectral stochastic Lagrangian approach, Comput. Methods Appl. Math. Eng. 195 (2006) 2289-2312.

[2] J. Aguado-Sierra, K.H. Parker, J.E. David, D. Francis, A.D. Hughes, J. Mayet, Arterial pulse wave velocity in coronary arteries, in: 28th International Conference of the Engineering in Medicine and Biology Society, 2006, IEEE-EMBS-2006.

[3] J. Alastruey, Numerical modelling of pulse wave propagation in the cardiovascular system: development, validation and clinical applications, PhD thesis, Imperial College London, University of London, UK, 2006.

[4] J. Alastruey, K.H. Parker, J. Peiró, S.M. Byrd, S.J. Sherwin, Modelling the Circle of Willis to assess the effects of anatomical variations and occlusions on Cerebral flows, J. Biomech. 40 (2007) 1794-1805.

[5] J. Alastruey, K.H. Parker, J. Peiró, S.J. Sherwin, Can the modified Allen's test always detect sufficient collateral flow in the hand? Comp. Meths. Biomech. Biomed. Eng. 9 (2006) 353-361.

[6] I. Babuška, F. Nobile, R. Tempone, A stochastic collocation method for elliptic partial differential equations with random input data, SIAM J. Numer. Anal. 45 (2007) 1005-1034. 
[7] I. Babuška, R. Tempone, G.E. Zouraris, Galerkin finite element approximations of stochastic elliptic differential equations, SIAM J. Numer. Anal. 42 (2004) 800-825.

[8] L. Euler, Principia pro motu sanguinis per arterias determinando, Opera posthima mathematica et physica anno 1844 detecta 2 (1775) 814-823 (Ediderunt PH Fuss et N Fuss Petropoli; Apund Eggers et Socios).

[9] G.S. Fishman, Monte Carlo: Concepts, Algorithms, and Applications, Springer-Verlag, New York, 1996.

[10] L. Formaggia, F. Nobile, A. Quarteroni, A one dimensional model for blood flow: application to vascular prosthesis, in: I. Babuška, T. Miyoshi, P.G. Ciarlet (Eds.), Mathematical Modeling and Numerical Simulation in Continuum Mechanics, Lecture Notes in Computational Science and Engineering, vol. 19, Springer-Verlag, Berlin, 2002, pp. 137-153.

[11] P. Frauenfelder, Ch. Schwab, R.A. Todor, Finite elements for elliptic problems with stochastic coefficients, Comput. Methods Appl. Mech. Eng. 194 (2005) 205-228.

[12] B. Ganapathysubramanian, N. Zabaras, Sparse grid collocation schemes for stochastic natural convection problems, J. Comput. Phys. (to appear).

[13] R. Ghanem, Hybrid stochastic finite elements: coupling of spectral expansions with Monte Carlo simulations, J. Appl. Mech. 65 (1998) 1004-1009.

[14] R.G. Ghanem, Ingredients for a general purpose stochastic finite element formulation, Comput. Methods Appl. Mech. Eng. 168 (1999) 19-34.

[15] R.G. Ghanem, R.M. Kruger, Numerical solution of spectral stochastic finite element systems, Comput. Methods Appl. Mech. Eng. 129 (1996) 289-303.

[16] R.G. Ghanem, J. Red-Horse, Propagation of uncertainty in complex physical systems using a stochastic finite elements approach, Physica D 133 (1999) 137-144.

[17] R.G. Ghanem, P. Spanos, Stochastic Finite Elements: A Spectral Approach, Springer-Verlag, 1991.

[18] D. Gottlieb, D. Xiu, Galerkin method for wave equations with uncertain coefficients, Commun. Comput. Phys. (submitted for publication).

[19] G.E. Karniadakis, S.J. Sherwin, Spectral/hp Element Methods for CFD, Oxford University Press, 2003.

[20] D.J. Kortweg, Uber die fortpflanzungesgechwindigkeit des schalles in elastischen rohern, Ann. Phys. Chem. (NS) 5 (1878) $525-527$.

[21] O. Le Maitre, O. Knio, H. Najm, R. Ghanem, Uncertainty propagation using Wiener-Haar expansions, J. Comput. Phys. 197 (2004) $28-57$.

[22] K.S. Matthys, J. Alastruey, J. Peiró, A.W. Khir, P. Segers, P.R. Verdonck, K.H. Parker, S.J. Sherwin, Comparison of a non-linear, one-dimensional numerical model with measurements in an in-vitro model of the human arterial system, J. Biomech. (submitted for publication).

[23] A.I. Moens, Over de voortplantingssnelheid von den pols (On the speed of propagation of the pulse), Technical Report, Leiden, 1877.

[24] M.S. Olufsen, Structured tree outflow condition for blood flow in larger systemic arteries, Am. J. Physiol. 276 (1999) H257H268.

[25] B. Riemann, Gesammelte mathematische werke und wissenschaftlicher nachlass, Technical Report, Gottingen, 1860.

[26] S.J. Sherwin, V.S. Franke, J. Peiró, K.H. Parker, One-dimensional modelling of a vascular network in space-time variables, J. Eng. Math. 47 (2003) 217-250.

[27] R. Skalak, The synthesis of a complete circulation, in: D. Bergel (Ed.), Cardiovascular Fluid Dynamics, vol. 2, Academic Press, London, 1972, pp. 341-376 (Chapter 2).

[28] S.A. Smolyak, Quadrature and interpolation formulas for tensor products of certain classes of functions, Soviet Math. Dokl. 4 (1963) $240-243$.

[29] N. Stergiopulos, D.F. Young, T.R. Rogge, Computer simulation of arterial flow with applications to arterial and aortic stenoses, J. Biomech. 25 (1992) 1477-1488.

[30] J.C. Stettler, P. Niederer, M. Anliker, Theoretical analysis of arterial hemodynamics including the influence of bifurcations. Part I: Mathematical model and prediction of normal pulse patterns, Ann. Biomed. Eng. 9 (1981) 145-164.

[31] J.C. Stettler, P. Niederer, M. Anliker, Theoretical analysis of arterial hemodynamics including the influence of bifurcations, part II: Critical evaluation of theoretical model and comparison with noninvasive measurements of flow patterns in normal and pathological cases, Ann. Biomed. Eng. 9 (1981) 165-175.

[32] M.A. Tatang, W.W. Pan, R.G. Prinn, G.J. McRae, An efficient method for parametric uncertainty analysis of numerical geophysical model, J. Geophys. Res. 102 (1997) 21925-21932.

[33] J.J. Wang, Wave propagation in a model of the human arterial system. PhD thesis, Imperial College, University of London, UK, 1997.

[34] J.J. Wang, K.H. Parker, Wave propagation in a model of the arterial circulation, J. Biomech. 37 (2004) 457-470.

[35] J.R. Womersley, An elastic tube theory of pulse transmission and oscillatory flow in mammalian arteries, Technical Report WADC Technical Report TR 56-614, Wright Air Development Center, 1957.

[36] D. Xiu, Efficient collocational approach for parametric uncertainty analysis, Commun. Comput. Phys. 2 (2) (2007) $293-309$.

[37] D. Xiu, J.S. Hesthaven, High-order collocation methods for differential equations with random inputs, SIAM J. Sci. Comput. 27 (3) (2005) 1118-1139.

[38] D. Xiu, G.E. Karniadakis, Modeling uncertainty in steady state diffusion problems via generalized polynomial chaos, Comput. Methods Appl. Math. Eng. 191 (2002) 4927-4948.

[39] D. Xiu, G.E. Karniadakis, The Wiener-Askey polynomial chaos for stochastic differential equations, SIAM J. Sci. Comput. 24 (2) (2002) 619-644. 
[40] D. Xiu, G.E. Karniadakis, Modeling uncertainty in flow simulations via generalized polynomial chaos, J. Comput. Phys. 187 (2003) 137-167.

[41] T. Young, Hydraulic investigations, subservient to an intended Croonian lecture on the motion of the blood, Phil. Trans. Roy. Soc. London 98 (1808) 164-186. 